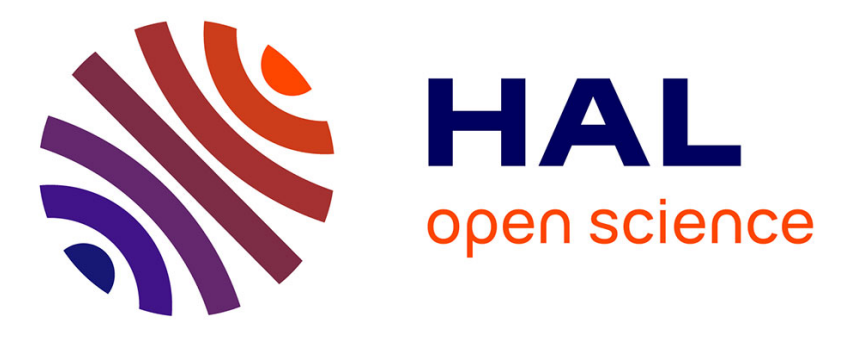

\title{
A 3D parallel boundary element method on unstructured triangular grids for fully nonlinear wave-body interactions
}

E. Dombre, J.C. Harris, Michel Benoit, D. Violeau, C. Peyrard

\section{- To cite this version:}

E. Dombre, J.C. Harris, Michel Benoit, D. Violeau, C. Peyrard. A 3D parallel boundary element method on unstructured triangular grids for fully nonlinear wave-body interactions. Ocean Engineering, 2019, 171, pp.505-518. 10.1016/j.oceaneng.2018.09.044 . hal-02121232

\section{HAL Id: hal-02121232 \\ https://hal.science/hal-02121232}

Submitted on 21 May 2020

HAL is a multi-disciplinary open access archive for the deposit and dissemination of scientific research documents, whether they are published or not. The documents may come from teaching and research institutions in France or abroad, or from public or private research centers.
L'archive ouverte pluridisciplinaire HAL, est destinée au dépôt et à la diffusion de documents scientifiques de niveau recherche, publiés ou non, émanant des établissements d'enseignement et de recherche français ou étrangers, des laboratoires publics ou privés. 
(C)2019. This manuscript version is made available under the CC-BY-NC-ND 4.0 license http://creativecommons.org/licenses/by-nc-nd/4.0

\title{
A 3D parallel boundary element method on unstructured triangular grids for fully nonlinear wave-body interactions
}

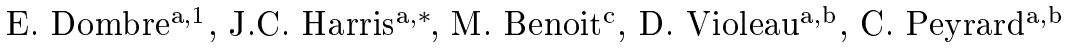 \\ ${ }^{a}$ LHSV, Ecole des Ponts, CEREMA, EDF RESD, Université Paris-Est, Chatou, France \\ ${ }^{b} E D F R \xi D$, Laboratoire National d'Hydraulique et Environnement, Chatou, France \\ ${ }^{c}$ Aix Marseille Univ., CNRS, Centrale Marseille, Institute de Recherche sur les Phénomènes Hors \\ Equilibre (IRPHE) UMR 7342, Marseille, France
}

\begin{abstract}
This paper presents the development and validation of a three-dimensional numerical wave tank devoted to studying wave-structure interaction problems. It is based on the fully nonlinear potential flow theory, here solved by a boundary element approach and using unstructured triangular meshes of the domain's boundaries. Time updating is based on a second-order explicit Taylor series expansion. The method is parallelized using the Message Passing Interface (MPI) in order to take advantage of multi-processor systems. For radiation problems, with cylindrical bodies moving in prescribed motion, the free-surface is updated with a fully Lagrangian scheme, and is able to reproduce reference results for nonlinear forces exerted on the moving body. For diffraction problems, semi-Lagrangian time-updating is used, and reproduces nonlinear effects for diffraction on monopiles. Finally, we study the nonlinear wave loads on a fixed semi-submersible structure, thereby illustrating the possibility to apply the proposed numerical model for the design of offshore structures and floaters.
\end{abstract}

Keywords: Nonlinear wave-structure interaction, Offshore structures, Ocean engineering, Boundary element method

\section{1. Introduction}

$2 \quad$ The numerical modeling of fully nonlinear interactions between floating structures and 3 waves in three dimensions (3D) is of great importance for the design of ocean engineering 4 structures such as offshore wind turbines or wave energy converters, as realistic sea states

5 may cause nonlinear motions of the structure. The problem is often addressed by means

- of the fully nonlinear potential flow (FNPF) approach, and has had broad success for

7 both radiation and diffraction problems.

\footnotetext{
* Corresponding author.

Email addresses: edombre@gmail.com (E. Dombre), jeffrey.harris@enpc.fr (J.C. Harris), benoit@irphe.univ-mrs.fr (M. Benoit), damien.violeau@edf.fr (D. Violeau), christ ophe.peyrard@edf.fr (C. Peyrard)

${ }^{1}$ Present address: Sogeti High Tech, 22-24 Rue du Gouverneur Général Éboué, 92130 Issy-lesMoulineaux.

Preprint submitted to Ocean Engineering
} 
Solving for FNPF involves the solution of Laplace's equation for a velocity potential, - which can be treated with the boundary element method (BEM). For one example, Ferrant 1 was able to get very good agreement for the loads on a vertical cylinder in regular waves, using linear triangular elements. More recently High Order Boundary Element Method (HOBEM) has seen more use, for its better properties in convergence, although it can be more complicated to produce an appropriate mesh for complex geometries.

Boo [2 studied the effect of linear and nonlinear irregular waves on a fixed bottom mounted cylinder with an HOBEM. In the work of Liu et al. [3], a HOBEM with structured meshes was used to compute the wave loads in forced motion and diffraction on a Wigley Hull and a truncated cylinder. A fair agreement with measurements performed at MARINTEK and the third order theory of Malenica and Molin [4, was found for the case of the truncated cylinder. Another HOBEM was recently developed by Bai and Eatock Taylor in [5] using unstructured grids and combined with a domain decomposition method in [6]. Various forced motions cases were investigated with a vertical cylinder. A remeshing step based on the Laplace smoothing technique was used. Typical nonlinear effects were outlined like the oscillation of the vertical force in surge at twice the frequency of the motion, occurring with symmetrical objects. An important nonlinear interaction between surge and pitch motion was also shown.

Other approaches have also been considered for these wave-body interaction problems with fully nonlinear potential flow. Ma et al. 7 developed an approach based on the finite element method (FEM) and applied it to wave loads on fixed vertical cylinders. Similar work with FEM was made by Wang et al. [8]. Shao and Faltinsen [9] developed the harmonic polynomial cell approach, which is another solution to potential flow problems based on a volume discretization, but representing the local solution as a linear superposition of elementary solutions of the Laplace equation, resulting in improvements in accuracy and speed. Mola et al. [10] used a BEM, but with substantial innovations with adaptive mesh refinement and stabilized by a Streamline Upwind Petrov-Galerkin (SUPG) scheme applied to the dominant transport term of the boundary condition, in case of a non-negligible advancing velocity.

While theoretically much of the physics is well understood even for cases with free motion, numerical complexities of working with higher-order methods mean that they are more difficult to work with in 3D for complex body shapes, hence even some recent works focus on 2D simulations, such as the FEM work by Yan and Ma [11] and by Wang and $\mathrm{Wu}[12$. Industrial design work can be done now though using such tools; the free motion of a simplified Floating Production Storage and Offloading (FPSO) structure was studied in [13]. Free motion was also addressed in [6, where the effect of the shape of the cylinder was investigated. An extension of the QALE-FEM method developed in [11 has also been applied to free motions in 14. Similar to the Laplace smoothing technique mentioned above used by Bai and Eatock Taylor [6], the QALE-FEM avoids remeshing of the free surface, but an adaptive mesh strategy is used based on a spring analogy for moving interior nodes of the free surface and the body surface. A validation of this scheme is performed with comparisons to experimental data for a barge-type and a spar-type floating structure with a good agreement.

Despite the many methods which exist, due to the numerous difficulties involved in fully nonlinear potential flow modeling, the state-of-the-art has not yet converged on a single approach, and industry models such as AEGIR 15 are normally used to only solve linear or second-order flow. 
where the function $c(\mathbf{x}, t)$ denotes the inner solid angle seen from the boundary (field) point $\mathbf{x}$, and $\mathbf{y}$ is taken to be the source point on the boundary, $\partial \Omega_{f}(t)$.

On the free surface $\Gamma_{f}(t)$, the kinematic and dynamic boundary conditions state that:

$$
\left\{\begin{array}{l}
\phi_{t}(\mathbf{x}, t)=-g z-\frac{1}{2} \nabla \phi(\mathbf{x}, t) \cdot \nabla \phi(\mathbf{x}, t) \text { for } \mathbf{x} \in \Gamma_{f}(t) \\
\frac{d \mathbf{x}}{d t}=\nabla \phi(\mathbf{x}, t) \text { for } \mathbf{x} \in \Gamma_{f}(t)
\end{array}\right.
$$

${ }_{85}$ The time-integration of these equations is described in the next section. 
On the solid boundary of the floating body $\Gamma_{c}(t)$, we specify a free-slip condition, which expresses the normal derivative of the potential equal to the normal component of the body velocity on that boundary:

$$
\phi_{n}(\mathbf{x}, t) \equiv \frac{\partial \phi}{\partial n}(\mathbf{x}, t)=\mathbf{v}_{b}(\mathbf{x}, t) \cdot \mathbf{n}_{b}(\mathbf{x}, t), \forall \mathbf{x} \in \Gamma_{f}(t)
$$

s6 where $\mathbf{n}_{b}(\mathbf{x}, t)$ denotes the unit normal vector pointing inward to the solid surface $\Gamma_{c}(t)$,

${ }_{87}$ at point $\mathbf{x}$, and $\mathbf{v}_{b}(\mathbf{x}, t)$ is the body velocity. This condition remains valid on the fixed

sв bottom and lateral boundaries, $\Gamma_{b} \cup \Gamma_{l}$, using a zero velocity, i.e., $\frac{\partial \phi}{\partial n}=0$.

To avoid the evaluation of the time derivative of the potential by use of a finite difference scheme, we apply the same BIE technique for computing $\phi_{t}$. Indeed $\phi_{t}$ satisfies the same field equation, and requires the associated boundary conditions. Following Dombre et al. [18], the Neumann boundary condition satisfied by $\phi_{t}$ on $\Gamma_{c}(t)$ is expressed as:

$$
\phi_{t n}(\mathbf{x}, t) \equiv \frac{\partial \phi_{t}}{\partial n}(\mathbf{x}, t)=\frac{d \mathbf{n}}{d t} \cdot\left(\mathbf{v}_{b}(\mathbf{x}, t)-\nabla \phi\right)+\mathbf{a}_{b}(\mathbf{x}, t) \cdot \mathbf{n}-\mathbf{v}_{b}(\mathbf{x}, t) \cdot(\nabla \nabla \phi \cdot \mathbf{n})
$$

s9 with $\mathbf{a}_{b}(\mathbf{x}, t)$ the solid acceleration vector at the position $\mathbf{x}$ and time $t$.

\section{эo 3. Numerical scheme}

\section{3.1. Boundary Element Discretization}

At each time-step, we solve the BIE problems associated to $\phi$ and to $\phi_{t}$ by using an isoparametric BEM with flat triangles. The whole set of boundaries of the domain is meshed with non-overlapping triangles. On each triangular element $\Gamma^{k}$, we assume the field variables and the geometry to have linear variations, described as:

$$
\left\{\begin{array}{l}
\phi^{k}\left(\xi_{1}, \xi_{2}\right)=\sum_{\left\{j ; \mathbf{x}_{j} \in \mathcal{V}^{k}\right\}} \phi_{j} N_{j}\left(\xi_{1}, \xi_{2}\right) \\
\mathbf{x}^{k}\left(\xi_{1}, \xi_{2}\right)=\sum_{\left\{j ; \mathbf{x}_{j} \in \mathcal{V}^{k}\right\}} \mathbf{x}_{j} N_{j}\left(\xi_{1}, \xi_{2}\right)
\end{array}\right.
$$

92 where $\mathcal{V}^{k}$ is the set of the vertices of $\Gamma^{k}$ and $\left(\xi_{1}, \xi_{2}\right)$ denotes the co-ordinates in the refer93 ence element $\Gamma_{\xi}$. The functions $N_{j}$ are the so-called shape functions, i.e., $N_{1}\left(\xi_{1}, \xi_{2}\right)=\xi_{1}$, ${ }_{94} \quad N_{2}\left(\xi_{1}, \xi_{2}\right)=\xi_{2}$, and $N_{3}\left(\xi_{1}, \xi_{2}\right)=1-\xi_{1}-\xi_{2}$.

Using a collocation method, we write that for any $\mathbf{x}_{i}$ belonging to the discrete boundary of the fluid domain $\Gamma_{j}=\partial \Omega_{j}$ at time $t_{j}$, we have:

$$
c\left(\mathbf{x}_{i}, t_{j}\right) \phi\left(\mathbf{x}_{i}, t_{j}\right)=\int_{\Gamma_{j}}\left(\phi_{n}\left(\mathbf{y}_{k}, t_{j}\right) G\left(\mathbf{y}_{k}, \mathbf{x}_{i}\right) d S_{k}-\phi\left(\mathbf{y}_{k}, t_{j}\right) \frac{\partial G}{\partial n}\left(\mathbf{y}_{k}, \mathbf{x}_{i}\right)\right) d S_{k}
$$

which can be, upon replacing the integral by a discrete sum over the vertices of the mesh, rewritten as:

$$
\left(\delta_{i j} c_{i}+\sum_{j=1}^{N_{d o f}} K_{i j}^{n}\right) \phi_{j}=\sum_{j=1}^{N_{\text {dof }}} K_{i j}^{d} \phi_{n}^{j}
$$


with $N_{d o f}$ the number of vertices of the mesh. Adopting notations used in [19], we can show that the sub-matrices of this system are defined as:

$$
\begin{aligned}
K_{i j}^{n} & =\sum_{k \in \mathcal{S}_{j}} \int_{\Gamma_{\xi}}\left(N_{l_{k}(j)}(\xi) \frac{\partial G}{\partial n}\left(\mathbf{x}^{k}(\xi), \mathbf{x}_{i}\right) J^{k}(\xi)\right) d \xi \\
K_{i j}^{d} & =\sum_{k \in \mathcal{S}_{j}} \int_{\Gamma_{\xi}}\left(N_{l_{k}(j)}(\xi) G\left(\mathbf{x}^{k}(\xi), \mathbf{x}_{i}\right) J^{k}(\xi)\right) d \xi
\end{aligned}
$$

95 where $l_{k}(j)$ is a local index varying from 1 to $3, \mathcal{S}_{j}$ denotes the set of elements containing

96 the node of global index $j$ and $J^{k}(\xi)$ is the Jacobian of the transformation which maps

${ }_{97} \Gamma_{\xi}$ to $\Gamma_{k}$. $l_{k}(j)$ thus associates to each $\Gamma_{k}$ in $\mathcal{S}_{j}$ the local index of the node $\mathbf{x}_{j}$ in

98 $\Gamma_{k}$. We also define the notation $S_{k}$ as the set of vertices belonging to the element $\Gamma_{k}$.

99 When assembling the matrix of the system (7), two situations may arise. If the source

100 point $\mathbf{x}_{p}$ is not belonging to the element $\Gamma^{k}$, both $K_{i j}^{d}$ and $K_{i j}^{n}$ are regular and we

101 use a quadrature formula defined by a Dunavant rule [20] in order to perform numerical

102 integration. Otherwise, the source point is belonging to $\Gamma^{k}$, i.e. there exists $\mathbf{x}_{j} \in S_{k}$ such

103 that $\mathbf{x}_{i}=\mathbf{x}_{j}$, in which case we treat the singularity by applying a Duffy transformation, similar to the polar change of coordinates presented in Grilli et al. [16].

In case of body motions, the velocity $\mathbf{v}_{b}$ is non zero and the present model requires to evaluate the high-order term $\mathbf{v}_{b} \cdot(\nabla \nabla \phi \cdot \mathbf{n})$ in Eq.(4), which is not trivial. This calculation may be simplified by transforming the second-derivative when computing the integral $\int_{\Gamma_{b}(t)} G \phi_{t n} d S_{y}$. This integral is decomposed into $I_{1}=\int_{\Gamma_{b}(t)} G \phi_{t n}^{(1)} d S_{y}$ and $I_{2}=\int_{\Gamma_{b}(t)} G \phi_{t n}^{(2)} d S_{y}$ with $\phi_{t n}^{(1)}=\frac{d \mathbf{n}}{d t} \cdot\left(\mathbf{v}_{b}-\nabla \phi\right)+\mathbf{a}_{b} \cdot \mathbf{n}$ and $\phi_{t n}^{(2)}=\mathbf{v}_{b} \cdot(\nabla \nabla \phi \cdot \mathbf{n})$. Following Bai and Teng [21], the use of the Stokes formula and basic vector analysis manipulations lead to the relationship:

$$
\begin{gathered}
I_{2}=\int_{\Gamma_{b}(t)} G\left(\mathbf{v}_{b} \cdot \nabla(\nabla \phi)\right) \cdot \mathbf{n} d S_{y}=\int_{\partial \Gamma_{b}(t)} G\left(\nabla \phi \times \mathbf{v}_{b}\right) \cdot d \mathbf{y}+ \\
\int_{\Gamma_{b}(t)} G\left[(\boldsymbol{\Omega} \times \nabla \phi)+(\nabla G \cdot \nabla \phi) \mathbf{v}_{b}-\left(\nabla G \cdot \mathbf{v}_{b}\right) \nabla \phi\right] \cdot \mathbf{n} d S_{y}
\end{gathered}
$$

assuming the rigid body velocity is given by $\mathbf{v}_{b}(\mathbf{x})=\mathbf{v}_{G}+\boldsymbol{\Omega} \times\left(\mathbf{x}-\mathbf{x}_{G}\right)$ with $\mathbf{v}_{G}$ the translational and $\boldsymbol{\Omega}$ the rotational velocity vectors of the rigid body. We recall that, by convention, the unit normal vector $\mathbf{n}$ is oriented towards the inside of the rigid body. In this respect, the line integral $\int_{\partial \Gamma_{b}(t)} G\left(\nabla \phi \times \mathbf{v}_{b}\right) \cdot d \mathbf{y}$ must be evaluated considering that the tangent vector to the waterline $d \mathbf{y}$ is such that the vector $d \mathbf{y} \times \mathbf{n}(\mathbf{y})$ points outside of the rigid body.

\subsection{Time-stepping}

As in the original 2D-FNPF code of [22], we update both the position and the potential on the free surface $\Gamma_{f}(t)$ by an explicit scheme based on a second-order explicit Taylor series expansion. In this scheme, the values of the potential $\phi$ and the position $\mathbf{x}$ at time 
$t^{i+1}$ may be expressed as follows:

$$
\left\{\begin{aligned}
\phi^{i+1} & =\phi\left(\mathbf{x}^{i+1}, t^{i+1}\right) \\
& =\phi\left(\mathbf{x}^{i}, t^{i}\right)+\frac{d \phi}{d t}\left(\mathbf{x}^{i}, t^{i}\right) \Delta t+\frac{d^{2} \phi}{d t^{2}}\left(\mathbf{x}^{i}, t^{i}\right) \frac{\Delta t^{2}}{2} \\
\mathbf{x}^{i+1} & =\mathbf{x}^{i}+\frac{d \mathbf{x}}{d t}\left(\mathbf{x}^{i}, t^{i}, \phi^{i}, \phi_{n}^{i}\right) \Delta t+\frac{d^{2} \mathbf{x}}{d t^{2}}\left(\mathbf{x}^{i}, t^{i}, \phi^{i}, \phi_{n}^{i}\right) \frac{\Delta t^{2}}{2}
\end{aligned}\right.
$$

From system (10), several numerical schemes can be devised according to the choice of the advection velocity used in the material derivative $\frac{d}{d t}$. Let $\mathbf{v}_{p}$ be a velocity field which is chosen to advect the free surface particles. When using the velocity $\mathbf{v}_{p}$ for moving the free surface nodes, we obtain the following first-order derivatives:

$$
\left\{\begin{array}{l}
\frac{d \phi}{d t}=\phi_{t}+\mathbf{v}_{p} \cdot \nabla \phi \\
\frac{d \mathbf{x}}{d t}=\mathbf{v}_{p}
\end{array}\right.
$$

Then, if we take again the material derivative of the first-order coefficients along the velocity vector $\mathbf{v}_{p}$, we obtain:

$$
\left\{\begin{array}{l}
\frac{d^{2} \mathbf{x}}{d t^{2}}=\frac{\partial \mathbf{v}_{p}}{\partial t}+\nabla \mathbf{v}_{p} \cdot \mathbf{v}_{p} \\
\frac{d^{2} \phi}{d t^{2}}=\frac{d \phi_{t}}{d t}+\frac{d \mathbf{v}_{p}}{d t} \cdot \nabla \phi+\mathbf{v}_{p} \cdot \frac{d \nabla \phi}{d t}
\end{array}\right.
$$

Given the free surface dynamic boundary condition $\phi_{t}=-g z-\frac{1}{2} \nabla \phi \cdot \nabla \phi$, we can derive:

$$
\frac{d \phi_{t}}{d t}=\mathbf{g} \cdot \mathbf{v}_{p}-\nabla \phi \cdot\left(\nabla \phi_{t}+\nabla \nabla \phi \cdot \mathbf{v}_{p}\right)
$$

We also have:

$$
\begin{aligned}
\frac{d \mathbf{v}_{p}}{d t} \cdot \nabla \phi+\mathbf{v}_{p} \cdot \frac{d \nabla \phi}{d t} & =\frac{\partial \mathbf{v}_{p}}{\partial t} \cdot \nabla \phi+\left(\nabla \mathbf{v}_{p} \cdot \mathbf{v}_{p}\right) \cdot \nabla \phi \\
& +\mathbf{v}_{p} \cdot \nabla \phi_{t}+\left(\nabla \nabla \phi \cdot \mathbf{v}_{p}\right) \cdot \mathbf{v}_{p}
\end{aligned}
$$

Summing all of these contributions, we obtain:

$$
\begin{aligned}
& \frac{d^{2} \phi}{d t^{2}}=\mathbf{g} \cdot \mathbf{v}_{p}+\left(\mathbf{v}_{p}-\nabla \phi\right) \cdot \nabla \phi_{t} \\
& +\frac{\partial \mathbf{v}_{p}}{\partial t} \cdot \nabla \phi+\left(\nabla \nabla \phi \cdot \mathbf{v}_{p}\right) \cdot\left(\mathbf{v}_{p}-\nabla \phi\right)+\left(\nabla \mathbf{v}_{p} \cdot \mathbf{v}_{p}\right) \cdot \nabla \phi
\end{aligned}
$$

One can easily check that setting $\mathbf{v}_{p}=\nabla \phi$ in Eq. (15) gives the Lagrangian second-order development:

$$
\frac{d^{2} \phi}{d t^{2}}=\mathbf{g} \cdot \nabla \phi+\nabla \phi_{t} \cdot \nabla \phi+(\nabla \nabla \phi \cdot \nabla \phi) \cdot \nabla \phi
$$

112 as can be found e.g. in Appendix A of [23]. 
We recall that the nonlinear conditions on the free surface read:

$$
\left\{\begin{array}{l}
\frac{\partial \eta}{\partial t}=\phi_{z}-\eta_{x} \phi_{x}-\eta_{y} \phi_{y} \\
\frac{\partial \phi}{\partial t}=-g \eta-\frac{1}{2} \nabla \phi \cdot \nabla \phi
\end{array}\right.
$$

${ }_{113}$ When dealing with fixed vertical cylinders or fully submerged bodies (or cases without ${ }_{114}$ any body), it can be useful to consider a semi-Lagrangian scheme, allowing only vertical 115 motion of the fluid particles. For this, we consider the definition $\mathbf{v}_{p}=\frac{\partial \eta}{\partial t} \mathbf{e}_{z}$. (For the 116 equivalent material derivative with curved structures, see Zhang and Kashiwagi [24].)

While expanding this scheme, we first make the assumption that the function $(x, y) \in$ $\mathbb{R}^{2} \mapsto \eta \in \mathbb{R}$ is single-valued (i.e., the waves are not overturning), which allows to get the relationships:

$$
\frac{\partial \eta}{\partial t}=\frac{\partial \phi}{\partial z}-\frac{\partial \eta}{\partial x} \frac{\partial \phi}{\partial x}-\frac{\partial \eta}{\partial y} \frac{\partial \phi}{\partial y}=\frac{\partial \phi}{\partial z}+\frac{n_{x}}{n_{z}} \frac{\partial \phi}{\partial x}+\frac{n_{y}}{n_{z}} \frac{\partial \phi}{\partial y}
$$

where $\mathbf{n}=\left(n_{x}, n_{y}, n_{z}\right)$ is the outward unit normal vector to the free surface. We can thus work out the following formula:

$$
\frac{\partial \eta}{\partial t}=\frac{\phi_{n}}{n_{z}}
$$

which is simpler to evaluate numerically as the present model relies on the distribution of the variables $\phi$ and $\phi_{n}$ on the boundary.

\subsubsection{Discrete velocity on unstructured grids}

For first-order elements, the velocity in a triangle is computed using a relationship found in Meyer et al. [25]. In the triangle number $j$, called $T_{j}$, the gradient of any linear function is calculated as follows:

$$
\begin{array}{r}
\nabla \phi_{T_{j}}=\frac{1}{2 A_{j}}\left(\left(\phi_{j, i+1}-\phi_{j, i}\right) \mathbf{n}_{j} \times\left(\mathbf{x}_{j, i}-\mathbf{x}_{j, i-1}\right)\right. \\
\left.+\left(\phi_{j, i-1}-\phi_{j, i}\right) \mathbf{n}_{j} \times\left(\mathbf{x}_{j, i+1}-\mathbf{x}_{j, i}\right)\right)
\end{array}
$$

where $A_{j}$ denotes the area of $T_{j}, \mathbf{n}_{j}$ the unit outward normal vector to $T_{j}$ and the index $(j, i)$ corresponds to the local index of the node number $i$ located on the triangle $T_{j}$. The indices $(j, i-1)$ and $(j, i+1)$ refer to local indices of the nodes in the triangle $T_{j}$ such that the arc $\overline{\mathbf{x}_{j, i-1} \mathbf{x}_{j, i} \mathbf{x}_{j, i+1}}$ is positively oriented with respect to the local normal vector $\mathbf{n}_{j}$. According to these definitions, we can further derive the following relationships:

$$
\begin{aligned}
& \mathbf{n}_{j}=\frac{\left(\mathbf{x}_{j, i+1}-\mathbf{x}_{j, i}\right) \times\left(\mathbf{x}_{j, i-1}-\mathbf{x}_{j, i}\right)}{\left\|\left(\mathbf{x}_{j, i+1}-\mathbf{x}_{j, i}\right) \times\left(\mathbf{x}_{j, i-1}-\mathbf{x}_{j, i}\right)\right\|} \\
& A_{j}=\frac{1}{2}\left\|\left(\mathbf{x}_{j, i+1}-\mathbf{x}_{j, i}\right) \times\left(\mathbf{x}_{j, i-1}-\mathbf{x}_{j, i}\right)\right\|
\end{aligned}
$$

We then take an average of Eq. (20) over the 1-ring neighborhood of the vertex $i$, which is the set of triangles containing $\mathbf{x}_{i}$ and denoted by $\mathcal{S}_{i}$ (see Fig. (1)). Taking into account 


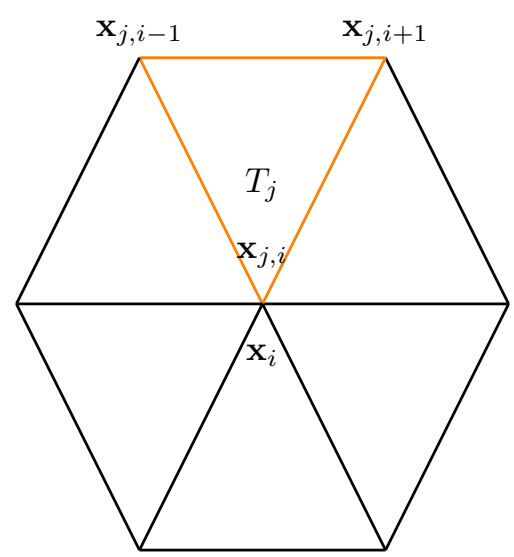

Figure 1: Sketch of the set of elements $S_{i}$, also called the 1-ring neighborhood of the vertex $\mathbf{x}_{i}$.

the contribution of $\phi_{n}$ to the gradient, we obtain an approximation of the velocity vector:

$$
\nabla \phi_{i}=\frac{1}{\sum_{j \in \mathcal{S}_{i}} A_{j}} \sum_{j \in \mathcal{S}_{i}} A_{j} \nabla \phi_{T_{j}}+\phi_{n} \frac{1}{\sum_{j \in \mathcal{S}_{i}} A_{j}} \sum_{j \in \mathcal{S}_{i}} A_{j} \mathbf{n}_{j}
$$

Eq. 200 has been, for example, used in the field of computer graphics [25] in order to evaluate geometric quantities such as the principal curvature on irregular meshes. It is shown in [25] to achieve a comparable accuracy to finite differences schemes and has the advantage to be less sensitive to the mesh configuration.

\subsubsection{Discrete acceleration on unstructured grids}

For evaluating the terms related to second-order derivatives of $\phi$, we try to take advantage of the previous methodology. This is made possible by expressing the secondorder tensor $\nabla \nabla \phi$ in a local basis attached to the vertex under consideration. In matrix form, this term reads:

$$
V_{k l}^{i}=\left(\nabla \nabla_{\mathcal{B}\left(\mathbf{x}_{\mathbf{i}}\right)} \phi\right)_{k l}=\nabla\left(\nabla \phi \cdot \mathbf{s}_{k}\right) \cdot \mathbf{s}_{l}
$$

with $\mathcal{B}\left(\mathbf{x}_{i}\right)=\left(\mathbf{s}_{1}, \mathbf{s}_{2}, \mathbf{s}_{3}\right)$ an orthonormal basis such that $\mathbf{s}_{3}$ is the normal vector to the discrete surface at the node $\mathbf{x}_{i}$. We define the vector:

$$
\begin{aligned}
\boldsymbol{\beta}_{i j k}=\frac{1}{2 A_{j}} & \left(\left(\nabla \phi_{j, i+1} \cdot \mathbf{s}_{k}-\nabla \phi_{j, i} \cdot \mathbf{s}_{k}\right) \mathbf{n}_{j} \times\left(\mathbf{x}_{j, i}-\mathbf{x}_{j, i-1}\right)\right. \\
& \left.+\left(\nabla \phi_{j, i-1} \cdot \mathbf{s}_{k}-\nabla \phi_{j, i} \cdot \mathbf{s}_{k}\right) \mathbf{n}_{j} \times\left(\mathbf{x}_{j, i+1}-\mathbf{x}_{j, i}\right)\right)
\end{aligned}
$$

The components of the matrix $V^{i}$ can be calculated as:

$$
\left\{\begin{array}{l}
V_{k l}^{i}=\frac{1}{\sum_{j \in \mathcal{S}_{i}} A_{j}}\left(\sum_{j \in \mathcal{S}_{i}} A_{j} \boldsymbol{\beta}_{i j k}\right) \cdot \mathbf{s}_{l}, \quad k \in\{1,2,3\}, l \in\{1,2\} \\
V_{13}^{i}=V_{31}^{i} \\
V_{23}^{i}=V_{32}^{i} \\
V_{33}^{i}=-V_{11}^{i}-V_{22}^{i}
\end{array}\right.
$$




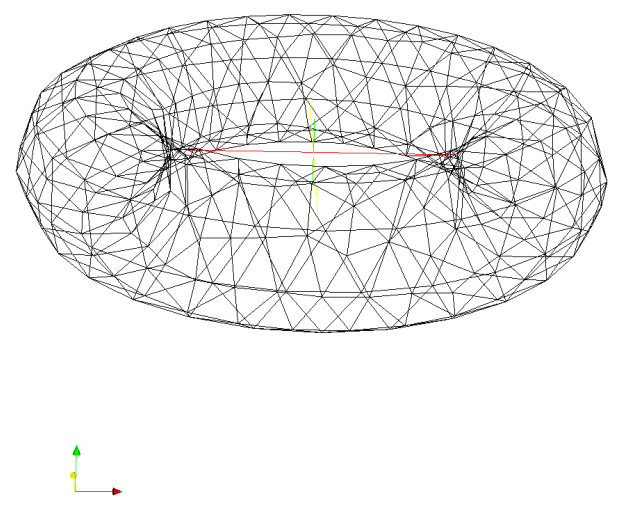

Figure 2: Irregular triangular grid of a torus with a mean radius of $R_{1}=2 \mathrm{~m}$ and a cross-section of radius $R_{2}=1 \mathrm{~m}$ generated by the algorithm NETGEN2D in the SALOME platform [26].

125 The relationships $V_{13}^{i}=V_{31}^{i}$ and $V_{23}^{i}=V_{32}^{i}$ are obtained by symmetry of the matrix $V^{i}$

This scheme consists therefore in interpolating linearly the projections of $\nabla \phi$ on the local basis $\mathcal{B}\left(\mathbf{x}_{i}\right)$ over the 1-ring neighbourhood of $\mathbf{x}_{i}$. The outward normal vector at $\mathbf{x}_{i}$ is evaluated by means of the following weighted sum:

$$
\mathbf{s}_{3}\left(\mathbf{x}_{i}\right)=\frac{\sum_{j \in \mathcal{S}_{i}} A_{j} \mathbf{n}_{j}}{\sum_{j \in \mathcal{S}_{i}} A_{j}}
$$

with the vector $\mathbf{n}_{j}$ previously defined on each triangle of $\mathcal{S}_{i}$. This vector is then scaled to define a unit normal vector, and from this we define two tangential vectors to the discrete surface at $\mathbf{x}_{i}$. For this purpose, we need to define a first tangential vector by projecting any point $\mathbf{x}_{j}$ belonging to a triangle of the set $\mathcal{S}_{i}$ and different from the point $\mathbf{x}_{i}$ onto the tangential plane oriented by $\mathbf{s}_{3}$ and passing through $\mathbf{x}_{i}$ by means of the following formula:

$$
\mathbf{s}_{1}\left(\mathbf{x}_{i}\right)=\frac{\mathbf{x}_{j}-\mathbf{x}_{i}-\mathbf{s}_{3}\left(\mathbf{x}_{i}\right) \cdot\left(\mathbf{x}_{j}-\mathbf{x}_{i}\right)}{\left\|\mathbf{x}_{j}-\mathbf{x}_{i}-\mathbf{s}_{3}\left(\mathbf{x}_{i}\right) \cdot\left(\mathbf{x}_{j}-\mathbf{x}_{i}\right)\right\|}
$$

A last vector of the basis $\mathcal{B}\left(\mathbf{x}_{i}\right)$ is then given by $\mathbf{s}_{2}\left(\mathbf{x}_{i}\right)=\mathbf{s}_{3}\left(\mathbf{x}_{i}\right) \times \mathbf{s}_{1}\left(\mathbf{x}_{i}\right)$. The performance of these discrete differential operators is finally assessed on the case of the irregular mesh of a torus with a mean radius of $R_{1}=2 \mathrm{~m}$ and a cross-section of radius $R_{2}=1 \mathrm{~m}$. An example of discretization for the torus is represented on Fig. 2. We use the function $\phi=\exp k_{z} z \sin \left(k_{x} x+k_{y} y\right)$ as a test case. We consider the following error indicators:

- The normalized maximum error of the vector $\nabla \phi$ (denoted by $L^{\infty}$ in the figures) and defined as $\epsilon=\frac{\max _{i=1 . . N}\left\|\nabla \phi-\nabla \phi_{\text {ref }}\right\|_{i}}{\max _{i=1 . . N}\left\|\nabla \phi_{\text {ref }}\right\|_{i}}$

- The normalized average error of the vector $\nabla \phi$ (denoted by $L^{1}$ in the figures) and defined as $\epsilon=\frac{\sum_{i=1}^{N}\left\|\nabla \phi-\nabla \phi_{\text {ref }}\right\|_{i}}{\sum_{i=1}^{N}\left\|\nabla \phi_{\text {ref }}\right\|_{i}}$ 

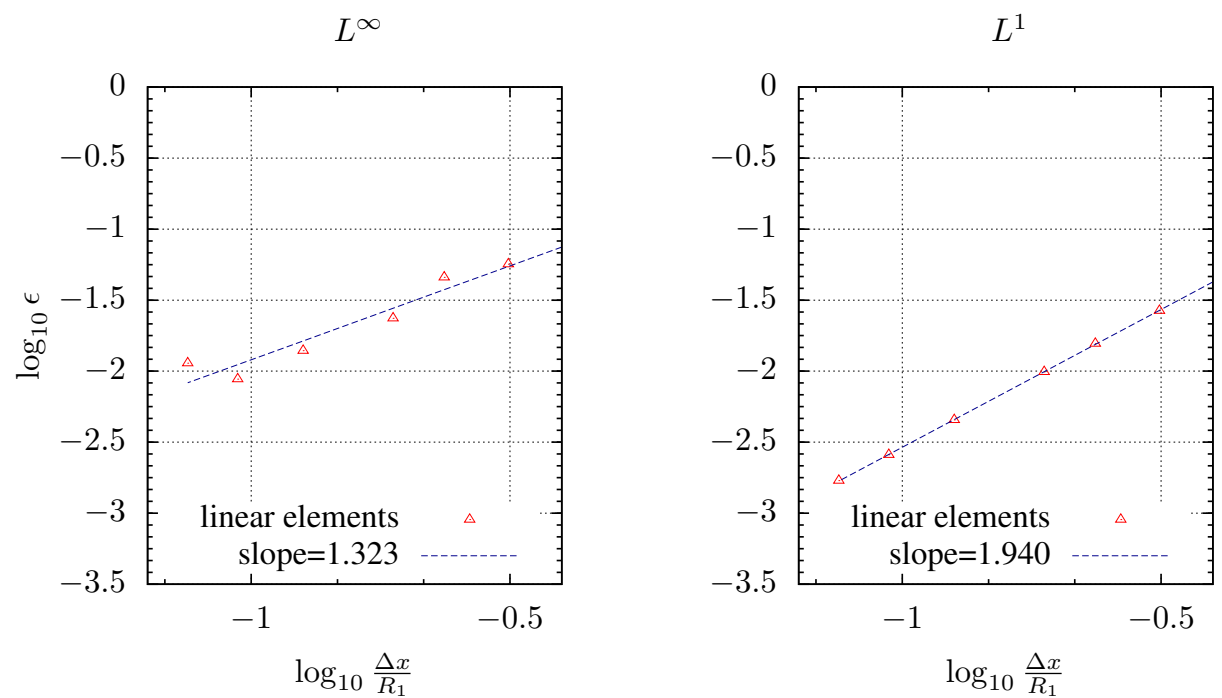

Figure 3: Maximum normalized error (left) and average normalized error (right) for the velocity vector $\mathbf{v}$ associated to the potential $\phi=\exp \left(k_{z} z\right) \sin \left(k_{x} x+k_{y} y\right)$ with $k_{x}=1 \mathrm{~m}^{-1}, k_{y}=0.5 \mathrm{~m}^{-1}$ and $k_{z}=$ $\sqrt{k_{x}^{2}+k_{y}^{2}}$ on a torus.
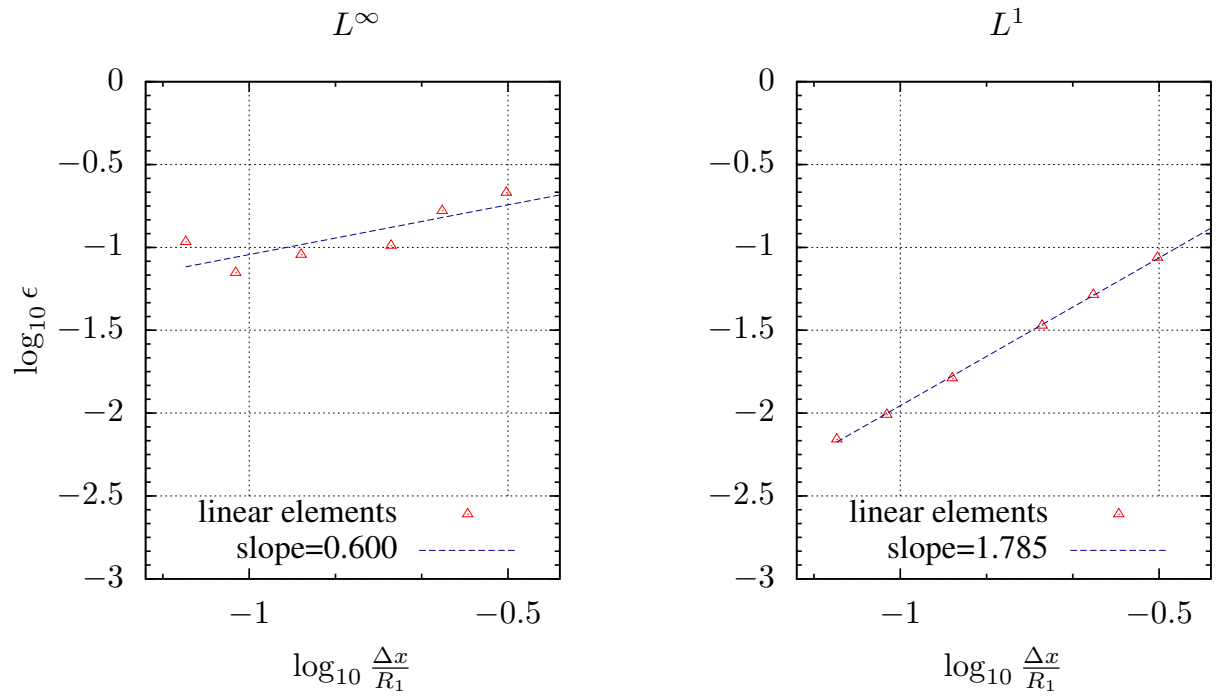

Figure 4: Maximum normalized error (left) and average normalized error (right) for the acceleration vector a associated to the potential $\phi=\exp \left(k_{z} z\right) \sin \left(k_{x} x+k_{y} y\right)$ with $k_{x}=1 \mathrm{~m}^{-1}, k_{y}=0.5 \mathrm{~m}^{-1}$ and $k_{z}=\sqrt{k_{x}^{2}+k_{y}^{2}}$ on a torus. 


\subsection{Mesh deformation}

\subsubsection{Time-updating of deformable surfaces}

The velocity potential $\phi$ and the position $\mathbf{x}$ on the free-surface are updated using Eq. (10). In order to avoid an incompatibility of velocity on the far-field edges, the freesurface boundary conditions are corrected in a certain area near the exterior boundary of the free surface. For a cylindrical fluid domain, as those which will be considered in the validation presented hereafter, we consider an inner cylindrical domain of radius $R_{\lambda}$ and an outer cylindrical domain of radius $R_{\text {ext }}$ (Fig. (6)). Then, the absorbing area is defined by the set $D_{\lambda}=\left\{\mathbf{x}=(r, \theta, z) \in \Gamma_{f}\right.$ such that $\left.(r, \theta) \in\left[R_{\lambda}, R_{e x t}\right] \times[0,2 \pi]\right\}$. In 


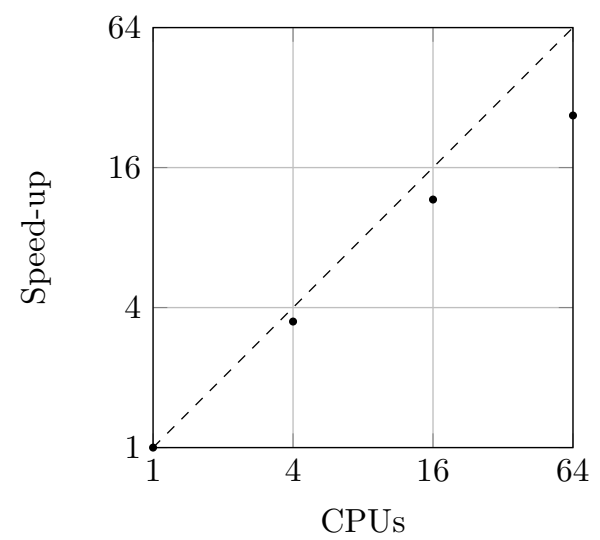

Figure 5: Speed-up $\frac{T_{n_{p}=1}}{T_{n_{p}}}$ of the assembling step plus the solution of the system matrix with respect to the number of processors $n_{p}$, invoked with MPI, with a mesh of 16,743 collocation nodes. $T_{n_{p}}$ is the $\mathrm{CPU}$ time of the process when using $n_{p}$ processors.

the set $D_{\lambda}$, the free surface and the potential are corrected as:

$$
\begin{aligned}
& z^{*} \leftarrow z-\Delta t \omega_{i}\left(\frac{r-R_{\lambda}}{R_{e x t}-R_{\lambda}}\right)^{2}\left(z-D(t) \eta_{i}(r, \theta, t)\right) \\
& \phi^{*} \leftarrow \phi-\Delta t \omega_{i}\left(\frac{r-R_{\lambda}}{R_{e x t}-R_{\lambda}}\right)^{2}\left(\phi-D(t) \phi_{i}\left(r, \theta, z^{*}, t\right)\right)
\end{aligned}
$$

where $\eta_{i}$ and $\phi_{i}$ correspond to a theoretical wave profile. For the absorption of the incident waves, other techniques like that developed by Clamond et al. [28] could be more efficient for irregular waves. The implementation of this method would require to solve (in parallel with MPI) an additional linear system corresponding to the vertices of the free surface mesh. This is left for future works.

For the bottom mounted cylinder, we will use a rectangular domain. In this case, the absorbing beach defined above is divided into two parts. The first part lies in front of the entrance of the tank and is defined as: $D_{\lambda, 1}=\left\{\mathbf{x}=\left(x_{1}, x_{2}, x_{3}\right) \in \Gamma_{f}\right.$ such that $\left(x_{1}, x_{2}\right) \in$ $\left.\left[0, L_{y}\right] \times\left[0, l_{\lambda}\right]\right\}$ where $l_{\lambda}$ is the length of the absorbing beach. In this domain, $\eta_{i}$ and $\phi_{i}$ will be chosen to correspond to a desired incident wave profile. At the end of the domain, a second beach is defined: $D_{\lambda, 2}=\left\{\mathbf{x}=\left(x_{1}, x_{2}, x_{3}\right) \in \Gamma_{f}\right.$ such that $\left(x_{1}, x_{2}\right) \in$ $\left.\left[0, L_{y}\right] \times\left[L_{x}-l_{\lambda}, L_{x}\right]\right\}$. In this second domain, we set $\eta_{i}=0$ and $\phi_{i}=0$.

\subsubsection{Time-updating of rigid surfaces}

The nodes associated to the fluid particles on the body surface are updated following the rigid body motion. We restrict ourselves to the forced motion of a rigid body. We recall that, with a rigid body, for any material points $\mathbf{x}_{A}$ and $\mathbf{x}_{B}$ belonging to the body, we have the relationship $\left\{\frac{d\left(\mathbf{x}_{B}-\mathbf{x}_{A}\right)}{d t}\right\}_{\mathcal{B}}=0$ in the reference frame of the body $\mathcal{B}$.

Rotation matrices around the axes of the fixed global reference frame are defined in the following fashion:

$$
R_{\theta_{x}}=\left(\begin{array}{ccc}
1 & 0 & 0 \\
0 & \cos \theta_{x} & -\sin \theta_{x} \\
0 & \sin \theta_{x} & \cos \theta_{x}
\end{array}\right)
$$




$$
\begin{aligned}
R_{\theta_{y}} & =\left(\begin{array}{ccc}
\cos \theta_{y} & 0 & \sin \theta_{y} \\
0 & 1 & 0 \\
-\sin \theta_{y} & 0 & \cos \theta_{y}
\end{array}\right) \\
R_{\theta_{z}} & =\left(\begin{array}{ccc}
\cos \theta_{z} & -\sin \theta_{z} & 0 \\
\sin \theta_{z} & \cos \theta_{z} & 0 \\
0 & 0 & 1
\end{array}\right)
\end{aligned}
$$

At any time, the link between the position expressed in $\mathcal{R}$ and the one expressed in $\mathcal{B}$ is made with the equation:

$$
\left(\mathbf{x}(t)-\mathbf{x}_{G}(t)\right)_{\mathcal{R}}=R_{\theta_{x}} R_{\theta_{y}} R_{\theta_{z}}\left(\mathbf{x}(t)-\mathbf{x}_{G}(t)\right)_{\mathcal{B}}
$$

with $\left(\mathbf{x}(t)-\mathbf{x}_{G}(t)\right)_{\mathcal{B}}$ a time-invariant vector. We define the fixed basis $\mathcal{R}=\left(\mathbf{e}_{x}, \mathbf{e}_{y}, \mathbf{e}_{z}\right)$ and the moving basis $\mathcal{B}=\left(\mathbf{e}_{x b}, \mathbf{e}_{y b}, \mathbf{e}_{z b}\right)$. The latter can be obtained by the transformation $R_{\theta_{x}} R_{\theta_{y}} R_{\theta_{z}}$ applied to the basis $\left(\mathbf{e}_{x}, \mathbf{e}_{y}, \mathbf{e}_{z}\right)$.

\subsubsection{Merging of the meshes}

As the free surface vertices are tracked in a Lagrangian manner during their motion, the meshes of the free-surface and the body need to be reconnected. Since the distance between the displaced waterline and the displaced body appears to be small, similarly to Bai and Eatock Taylor [5, we make a projection of the nodes located on the waterline onto the body geometry. Here we consider an application of this technique in the case of a cylindrical body surface. The following procedure is used for each node of the waterline:

1. Apply the MEL time-stepping to the position $\mathbf{x}_{f}^{*}$ :

$$
\mathbf{x}_{f}^{*}(t+\Delta t)=\mathbf{x}_{f}(t)+\frac{d \mathbf{x}_{f}}{d t} \Delta t+\frac{d^{2} \mathbf{x}_{f}}{d t^{2}} \frac{\Delta t^{2}}{2}
$$

2. Express the vector $\mathbf{x}_{f}^{*}$ in the body reference frame $\mathcal{B}$ :

$$
\begin{aligned}
& \quad \mathbf{x}_{f}^{*}(t+\Delta t)_{\mathcal{B}}=\mathbf{x}_{G}(t+\Delta t)_{\mathcal{B}} \\
& +R_{\theta_{z}}^{-1} R_{\theta_{y}}^{-1} R_{\theta_{x}}^{-1}\left(\mathbf{x}_{f}^{*}(t+\Delta t)-\mathbf{x}_{G}(t+\Delta t)\right)_{\mathcal{R}}
\end{aligned}
$$

3. Express $\mathbf{x}_{f}^{*}(t+\Delta t)_{\mathcal{B}}$ in a coordinate system suited to the shape under consideration (for a cylinder, we choose the polar coordinate system):

$$
\begin{aligned}
& \mathbf{x}_{f}^{*}(t+\Delta t)_{\mathcal{B}}=\mathbf{x}_{G}(t+\Delta t)_{\mathcal{B}} \\
& +r_{f}\left(\cos \theta_{f} \mathbf{e}_{x b}+\sin \theta_{f} \mathbf{e}_{y b}\right)+z_{f} \mathbf{e}_{z b}
\end{aligned}
$$

4. Replace the radial coordinate $r_{f}$ by the radius of the cylinder $R_{b}$ in the reference frame $\mathcal{B}$ :

$$
\begin{aligned}
& \mathbf{x}_{f}(t+\Delta t)_{\mathcal{B}}=\mathbf{x}_{G}(t+\Delta t)_{\mathcal{B}} \\
& +R_{b}\left(\cos \theta_{f} \mathbf{e}_{x b}+\sin \theta_{f} \mathbf{e}_{y b}\right)+z_{f} \mathbf{e}_{z b}
\end{aligned}
$$

5. Transform the new coordinate $\mathbf{x}_{f}(t+\Delta t)_{\mathcal{B}}$ into the global reference frame $\mathcal{R}$ :

$$
\begin{aligned}
& \mathbf{x}_{f}(t+\Delta t)_{\mathcal{R}}=\mathbf{x}_{G}(t+\Delta t)_{\mathcal{R}} \\
& +R_{\theta_{x}} R_{\theta_{y}} R_{\theta_{z}}\left(\mathbf{x}_{f}(t+\Delta t)-\mathbf{x}_{G}(t+\Delta t)\right)_{\mathcal{B}} \\
& 13
\end{aligned}
$$


For each geometric node of the waterline, we denote by $\mathbf{x}_{f}$ the position vector of the node belonging to the mesh of the free surface and by $\mathbf{x}_{b}$ the position vector of the node belonging to the mesh of the body surface. Prior to updating the position of the nodes for the next time-step, $\mathbf{x}_{f}$ and $\mathbf{x}_{b}$ are equal. An interpolation of the free surface elevation angular distribution found with the procedure described above, is made on a fixed uniform angular distribution. In other words, for each double-node of the intersection, we first express $\mathbf{x}_{f}$ and $\mathbf{x}_{b}$ in the basis $\left(\mathbf{e}_{x b}, \mathbf{e}_{y b}, \mathbf{e}_{z b}\right)$ :

$$
\begin{aligned}
\left(\mathbf{x}_{f}-\mathbf{x}_{G}\right)_{\mathcal{B}} & =R_{b}\left(\cos \theta_{f} \mathbf{e}_{x b}+\sin \theta_{f} \mathbf{e}_{y b}\right)+z_{f} \mathbf{e}_{z b} \\
\left(\mathbf{x}_{b}-\mathbf{x}_{G}\right)_{\mathcal{B}} & =R_{b}\left(\cos \theta_{b} \mathbf{e}_{x b}+\sin \theta_{b} \mathbf{e}_{y b}\right)+z_{f} \mathbf{e}_{z b}
\end{aligned}
$$

Then, for each angle $\theta_{b j}$, we compute the indices:

$$
\begin{aligned}
& i_{1}=\operatorname{argmin}_{i \in \llbracket 1, N_{d} \rrbracket}\left[\left|\theta_{f i}-\theta_{b j}\right| ; \theta_{b j} \geq \theta_{f i}\right] \\
& i_{2}=\operatorname{argmin}_{i \in \llbracket 1, N_{d} \rrbracket}\left[\left|\theta_{f i}-\theta_{b j}\right| ; \theta_{b j}<\theta_{f i}\right]
\end{aligned}
$$

with $N_{d}$ the number of double-nodes at the intersection of the mesh of the body surface and the mesh of the free surface. Afterwards, we interpolate linearly the values of the potential $\phi_{j}$ and the values of the local free-surface elevation $\left(\mathbf{x}_{f}-\mathbf{x}_{b}\right)_{\mathcal{B}} \cdot \mathbf{e}_{z b}$ in the interval $\left[\theta_{i_{1}}, \theta_{i_{2}}\right]$.

\subsubsection{Filtering of the waterline}

When considering steep sea states, one often observes instabilities near the waterline, which eventually propagate throughout the domain. As in many publications, we thus filter, at each time step, the variables of the waterline defined previously. We apply, similarly to [29, 30, a filtering formula with a moving stencil of 7 points for the variables $\phi$ and $\left(\mathbf{x}_{f}-\mathbf{x}_{b}\right)_{\mathcal{B}} \cdot \mathbf{e}_{z b}$ along the waterline only. For the potential $\phi$, this formula reads:

$$
\phi_{i}=\frac{1}{32}\left(-\phi_{i-3}+9 \phi_{i-1}+16 \phi_{i}+9 \phi_{i+1}-\phi_{i+3}\right) .
$$

\subsubsection{Remeshing step}

In time-domain simulations with floating bodies, the geometry is always changing. As a consequence it is necessary to change the mesh of the geometry. On the one hand, in order to prevent the triangles to be distorted in the vicinity of the piercing surface cylinder, a Laplace smoothing technique is applied, for the mesh of the free surface, on the projection of the nodes onto an horizontal plane. This technique has also been used by [7, 5. On the other hand, the mesh of the body is generated with regular quadrangles divided into triangles by their diagonal. For this part of the mesh, we modify only the vertical position of the nodes to get a uniform vertical distribution.

\section{Numerical results}

\subsection{Sway motion of a truncated vertical cylinder \\ 4.1.1. Problem setup}

We are concerned in this section with the imposed periodic motion in sway (i.e. along $x_{1}$ axis) of a truncated cylinder. The position vector of the center of gravity is given the form:

$$
\mathbf{x}_{G}(t)=\left(x_{1}(t), x_{2}(t), x_{3}(t)\right)=(A \sin \omega t, 0,0)
$$




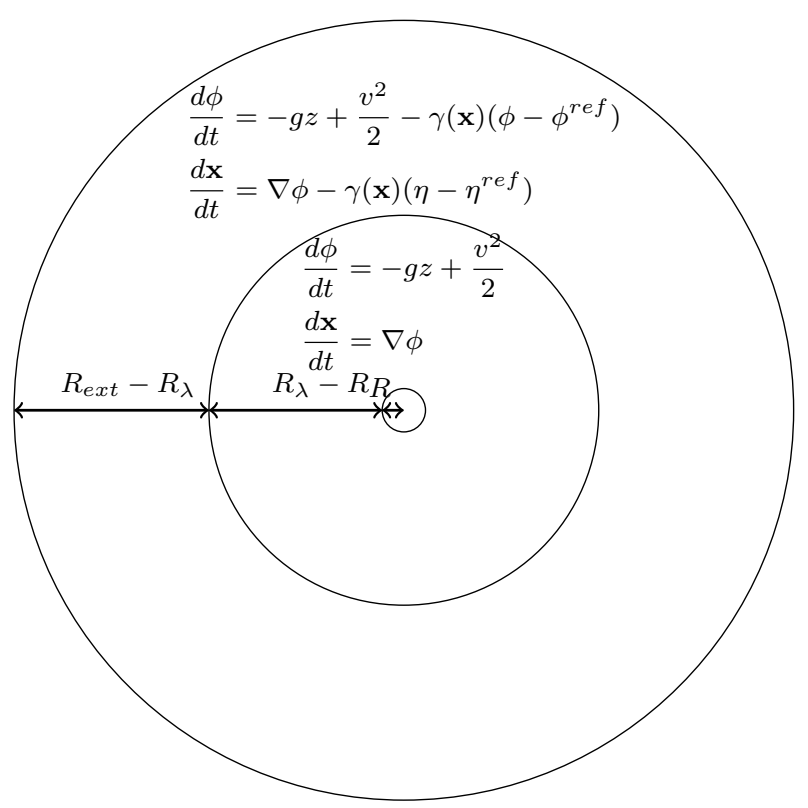

Figure 6: Sketch of the decomposition of the domain with respect to the free surface boundary conditions, noting boundary conditions for fully Lagrangian time-updating. In this case, Laplace smoothing is used to maintain a well formed mesh on the free-surface.

with $\omega$ the angular frequency of motion. The water depth of the numerical wave tank is set to $d=1.5 R$, with $R$ the radius of the cylinder, and the draft of the cylinder is set to $B=0.5 R$. The shape of the fluid domain is also cylindrical, with a radius $R_{e x t}$. Moreover, the fluid domain is divided into two cylindrical parts, as represented on Fig. (6).

An outer sub-domain is defined, where the boundary conditions include corrective terms, e.g., $\gamma(\mathbf{x})\left(\eta-\eta^{r e f}\right)$ for the free surface elevation, with $\eta^{r e f}$ an analytical free surface elevation and $\gamma$, a space-dependent factor. For the radiation, $\eta^{r e f}=0$ and $\phi^{r e f}=0$ in order to damp the reflected waves radiated by the body motion. In the inner domain, the fully nonlinear boundary conditions are used for determining the evolution of the free surface elevation and potential. The radius of the inner cylinder is denoted by $R_{\lambda}$. Such a configuration has been employed for instance by Ferrant [31.

\subsubsection{Mesh convergence}

In this section, we study the mesh convergence of the model for one of the frequencies tested hereafter. We note that by having a damping region such as Eq. 28, as used before by Cointe [32], volume conservation is not inherently guaranteed, as the freesurface boundary conditions are modified. We consider the non-dimensional wavenumber $k R=1.4$ (with a cylinder radius of $1 \mathrm{~m}$ ), which gives a wavelength $\lambda \approx 4.48 \mathrm{~m}$ and a period $T \approx 1.72 \mathrm{~s}$. The radius of the external domain is chosen as $R_{e x t}=8 \mathrm{~m}$. An absorbing beach of radial length $4.5 \mathrm{~m}$ is used. We check in this section the convergence by looking at the conservation of the discrete water volume in the wave tank. The characteristics of the meshes used for the convergence study are recalled in Table 1 . 


\begin{tabular}{l|ccccc}
\hline & Mesh $a$ & Mesh $b$ & Mesh $c$ & Mesh $d$ & Mesh $e$ \\
\hline \hline$\frac{2 \pi R_{\text {ext }}}{\Delta r_{e}}$ & 100 & 150 & 200 & 250 & 300 \\
\hline$\frac{2 \pi R}{\Delta r_{e}}$ & 20 & 30 & 40 & 50 & 60 \\
\hline$\frac{h}{\Delta z_{e}}$ & 4 & 6 & 6 & 8 & 10 \\
\hline$\frac{B}{\Delta z_{i}}$ & 8 & 12 & 14 & 18 & 20 \\
\hline $\min l_{1 D}[\mathrm{~m}]$ & 0.313 & 0.209 & 0.157 & 0.126 & 0.105 \\
\hline $\max l_{1 D}[\mathrm{~m}]$ & 0.505 & 0.335 & 0.251 & 0.201 & 0.168 \\
\hline$N_{F S}$ & 856 & 3141 & 3286 & 12437 & 12576 \\
\hline$N_{\text {body }}$ & 210 & 535 & 760 & 1116 & 1940
\end{tabular}

Table 1: Truncated cylinder in sway motion along the axis $O x$ : characteristics of the meshes used in the volume conservation study. $\Delta_{r_{e}}$ (respectively $\Delta_{r_{i}}$ ) is the space-step of the mesh on the circumference of the outer (respectively inner) cylinder. $\Delta_{z_{e}}$ (respectively $\Delta_{z_{i}}$ ) is the space-step of the mesh on the vertical direction of the outer (respectively inner) cylinder. $N_{F S}$ is the number of nodes on the free surface. $N_{b o d y}$ is the number of nodes on the truncated cylinder surface. $l_{1 D}$ is the length of the edges on the mesh of the free surface.

In Fig. 7. we represent the volume error defined as:

$$
\epsilon\left(t^{+}\right)=\frac{V\left(t^{+}\right)-V\left(t^{+}=0\right)}{V\left(t^{+}=0\right)}
$$

as a function of the non-dimensional time $t^{+}=\frac{t}{T}$ and the spatial discretization. For each mesh, we enforce a Courant-Friedrichs-Lewy (CFL) condition by automatically adapting the time-step as follows:

$$
\Delta t=\mathcal{C}_{0} \frac{\min _{(i, j) ; i<j ;\left(\mathbf{x}_{i}, \mathbf{x}_{j}\right) \in \Gamma_{f}(t)^{2}}\left\|\mathbf{x}_{i}-\mathbf{x}_{j}\right\|}{\sqrt{g d}}
$$
where we choose $\mathcal{C}_{0}=0.4$, following Grilli et al. 33 .

It is seen in Fig. 7 that the error $\epsilon_{V}$ stabilizes after 6 periods and decreases with the time-step. More specifically, after 7 periods, the mean relative error is around $8.10^{-5}$ for the time-step $\Delta t_{a}=0.0122 T$, while it is around $4.10^{-5}$ for the time-step $\Delta t_{b}=$ $0.0069 T \approx \frac{1}{2} \Delta t_{a}$. This shows that the error is decreasing linearly with the time-step. Moreover we can observe that the amplitude of the high frequency oscillations decreases also with the time-step.

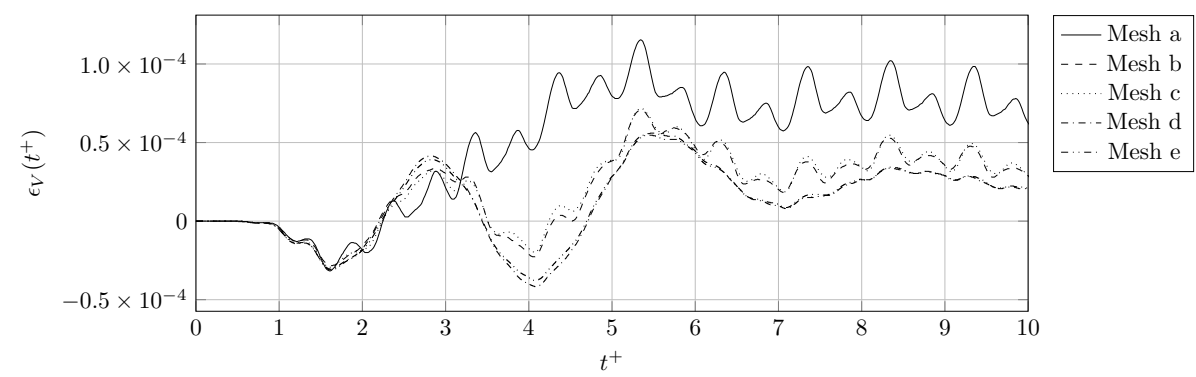

Figure 7: Truncated cylinder in sway motion along the axis $O x$ : computation of the relative volume error $\epsilon_{V}\left(t^{+}\right)$for various spatial and time discretizations. 


\subsubsection{Force coefficients}

The radius of the outer cylinder is set to $R_{e x t}=2 \lambda$ where $\lambda$ is the wavelength expected from the linear theory at the water depth $d$ and the angular frequency $\omega$ (Fig. 8). The entrance of the absorbing beach is located at a distance $R_{\lambda}=\lambda$ from the origin $(0,0,0)$. This problem has been recently addressed by Zhou et al. [29], using a HOBEM devised for structured quadrangular meshes. Our results are compared to the linear BEM of Yeung [34, the second-order frequency BEM model of Teng et al. [35, and the nonlinear method of Zhou et al.

For the amplitude of motion under consideration, $\frac{A}{R}=0.15$, we observe on Fig. (9) a fair agreement between the added-mass computed with our model and the results of Zhou et al. [29]. The discrepancy with the linear results of Yeung is reduced as the nondimensional wavenumber $k R$ decreases. This behavior is not surprising as, for a given choice of non-dimensional amplitude $\frac{A}{R}$, the associated wave steepness will increase with increasing value of $k R$, resulting in larger nonlinear effects.

For the linear radiation damping coefficient $B_{11}$, represented in Fig. 10 a still closer agreement with the linear theory is found. A similar agreement is observed for the nondimensional first-order coefficient of the horizontal force $\frac{F_{x}^{(1)}}{\rho A R^{2}}$, represented on Fig. 11 except a slight discrepancy which appears at $k R=1$.

Despite the apparently linear behavior of the horizontal force, this case clearly shows the interest of using a nonlinear model. As mentioned by Zhou et al. [29, according to a theoretical result demonstrated by $\mathrm{Wu}[36$, the vertical force oscillates at twice the frequency of the motion $2 \omega$. As before, the discrepancy between the weakly nonlinear model of Teng et al. [35] and the fully nonlinear model of Zhou et al. increases with increasing values of the parameter $k R$. The existence of the second-order coefficient $F_{z}^{(2)}$ is well captured with our model as can be seen in Fig. 12. We see that our method allows us to find a very good agreement with the frequency analysis, for wavenumbers such that $k R \leq 0.8$. For decreasing values of $k R$, the present results exhibit a closer convergence towards the results of the second-order model in comparison with the model of Zhou et $a l$. For larger $k R$, our nonlinear model deviates from the weakly nonlinear model and predicts higher values of $F_{z}^{(2)}$, which is not the case of the model of Zhou et al.
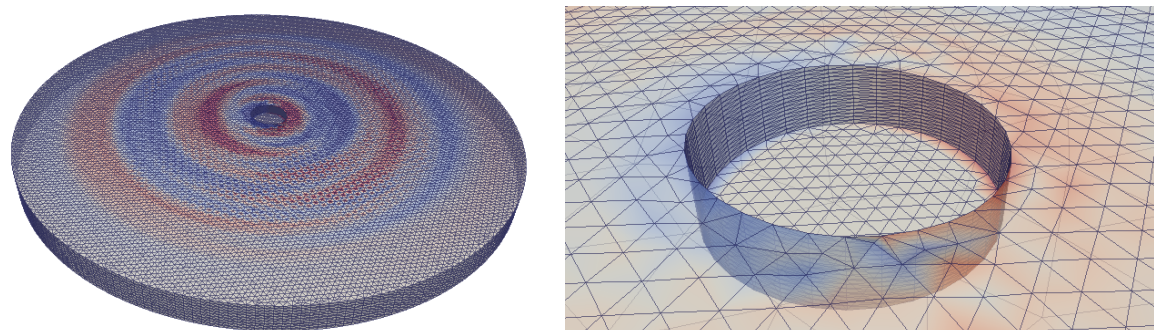

Figure 8: Computational domain (left panel) and close-up of grid near cylinder (right panel) for test cases of waves moving cylinder. 


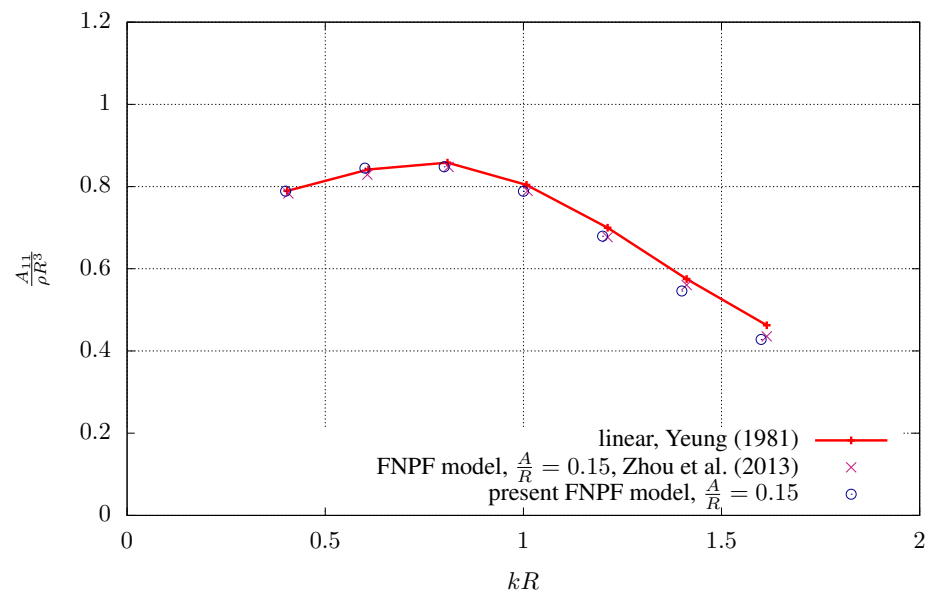

Figure 9: Truncated cylinder in sway motion along the axis $O x$ : computation of the added-mass with respect to the non-dimensional wavenumber $k R$ for various numerical models.

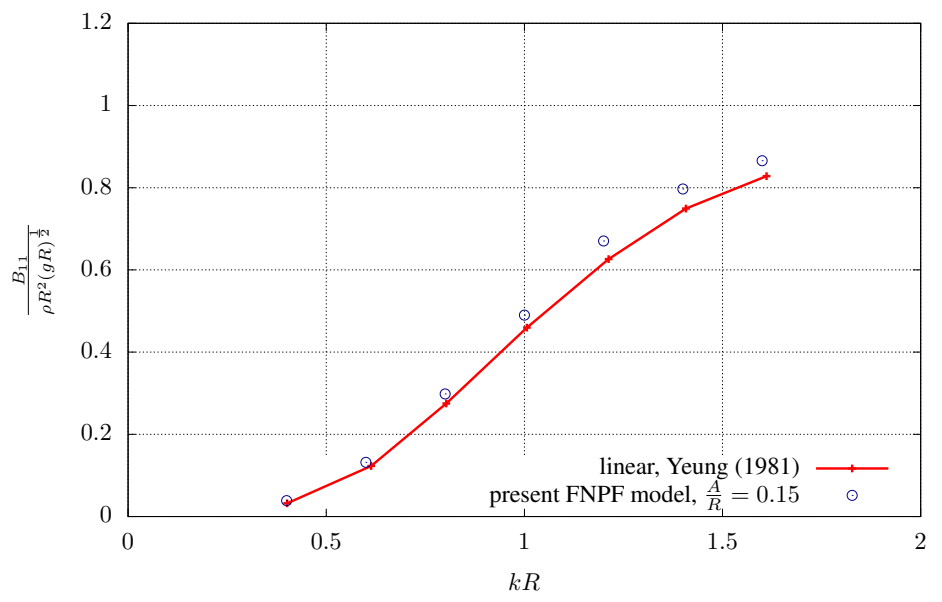

Figure 10: Truncated cylinder in sway motion along the axis $O x$ : computation of the radiation damping coefficient with respect to the non-dimensional wavenumber $k R$ for various numerical models. 


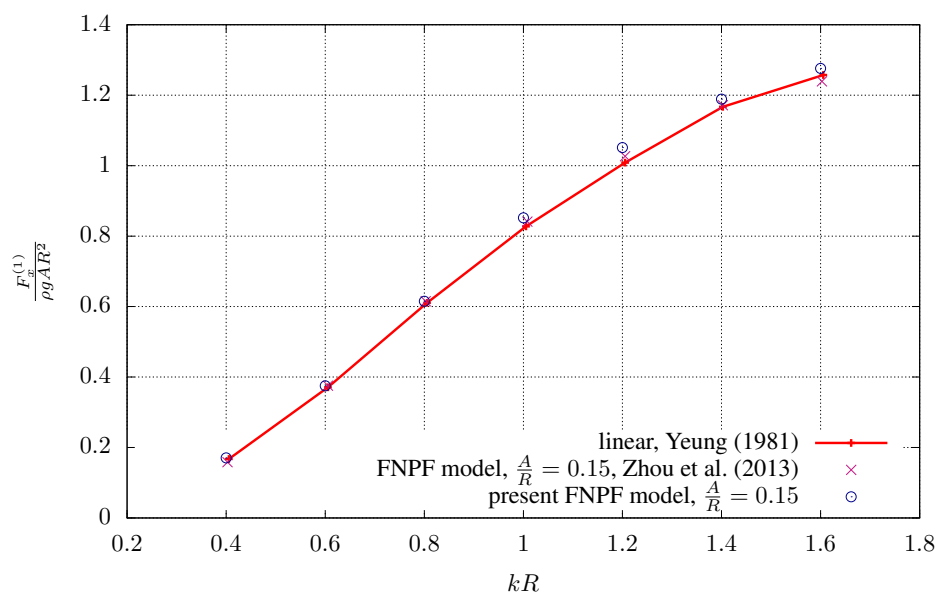

Figure 11: Truncated cylinder in sway motion along the axis $O x$ : first-order Fourier coefficient associated to the horizontal force $F_{x}$ with respect to the non-dimensional wavenumber $k R$ for various numerical models.

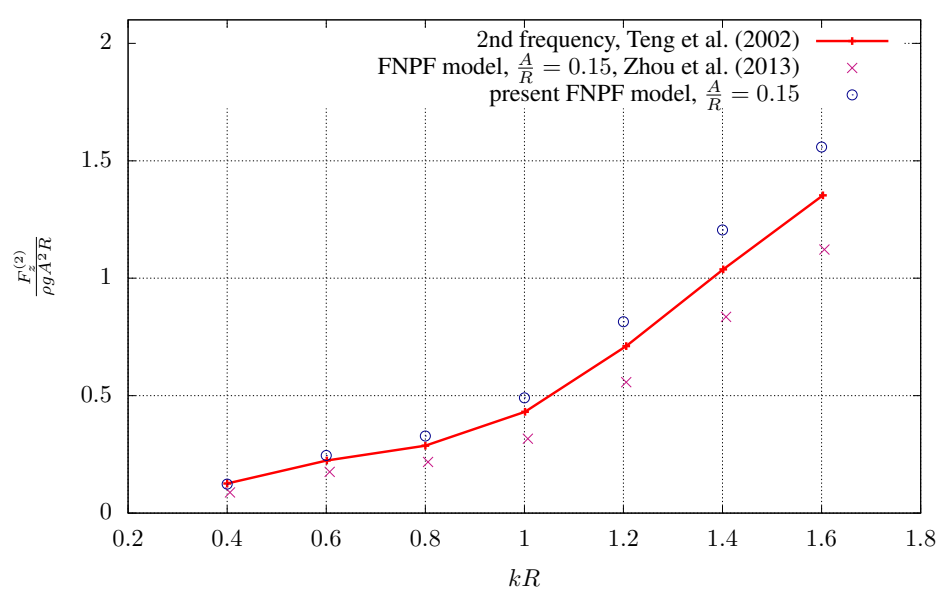

Figure 12: Truncated cylinder in sway motion along the axis $O x$ : second-order Fourier coefficient associated to the vertical force $F_{z}$ with respect to the non-dimensional wavenumber $k R$ for various numerical models. 


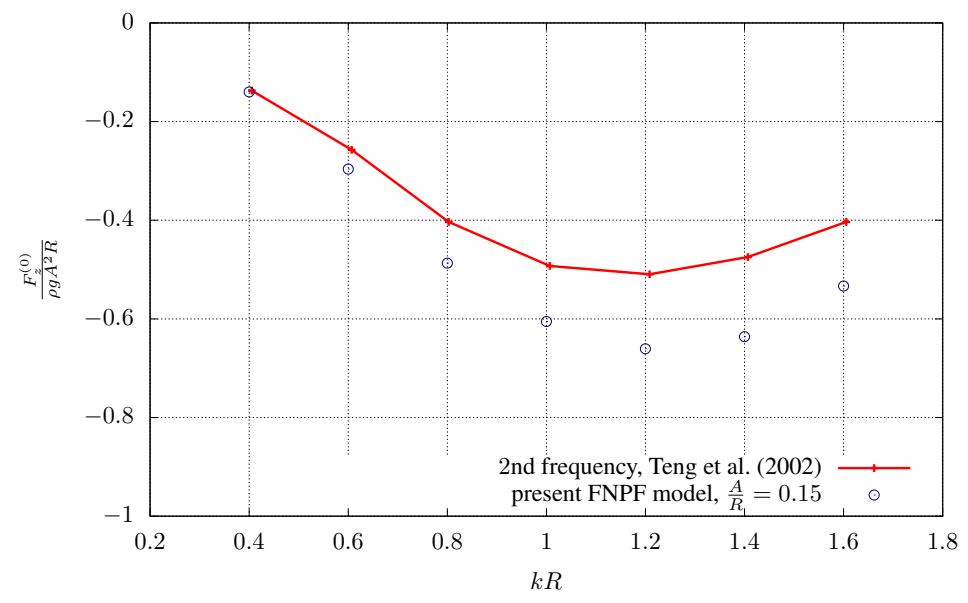

Figure 13: Truncated cylinder in sway motion along the axis $O x$ : zero-order Fourier coefficient associated to the vertical force $F_{z}$ with respect to the non-dimensional wavenumber $k R$ for various numerical models.

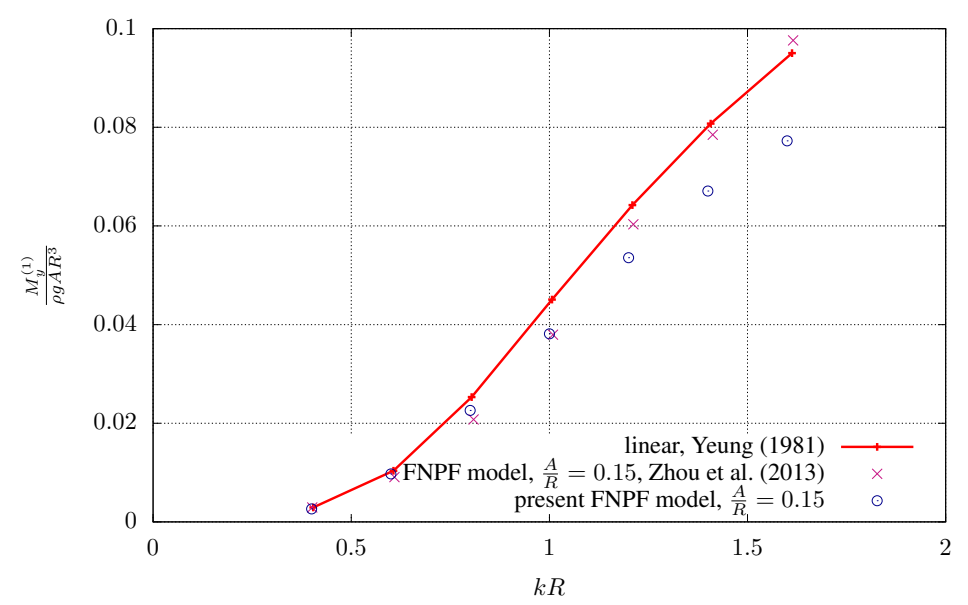

Figure 14: Truncated cylinder in sway motion along the axis $O x$ : first-order Fourier coefficient associated to the overturning moment $M_{y}$ with respect to the non-dimensional wavenumber $k R$ for various numerical models. 
On Fig. 13 the non-dimensional difference between the zero-order coefficient $F_{z}^{(0)}$ and the hydrostatic force $\rho g V_{b}, V_{b}$ being the discretized wet volume of the body, is represented. Our method predicts very well the load for $k R=0.4$ and the trend of this quantity is correctly reproduced.

Finally, we represent on Fig. 14 the variation of the first-order coefficient associated to the overturning moment $\frac{M_{y}^{(1)}}{\rho A R^{3}}$. Our results are very close to those of Zhou et al. [29] for wavenumbers $k R$ smaller than $k R=1$. For larger values of $k R$, we observe with our model a clear deviation from the linear theory, suggesting a significant influence of nonlinear effects at high frequencies, although this is contrary to the results of Zhou et al..

\subsection{Diffraction of long waves on a bottom-mounted cylinder}

In this section, we analyze the diffraction of a long wave on a bottom mounted vertical cylinder of radius $R=0.25 \mathrm{~m}$ (Fig. 15 ). The wavenumber $k$ is chosen such that $k R=$ 0.245 , which gives for linear theory a wave period $T=2.03 \mathrm{~s}$. Simulations are repeated for 6 different wave amplitudes such that $k A=\{0.025,0.05,0.075,0.10,0.125,0.150\}$. The signal is analyzed with a discrete Fast Fourier Transform on the time-interval $t^{+}=$ $\frac{t}{T} \in[5,13]$. For this case, the wavelength in deep-water may be estimated as $\lambda \approx 6.41 \mathrm{~m}$. The domain is a box with the dimensions $L_{x} \times L_{y} \times L_{z}=26 \mathrm{~m} \times 6 \mathrm{~m} \times 3.2 \mathrm{~m}$. The mesh used for that study contains $N_{d o f}=16,743$ nodes. When generating the mesh of the free surface with the algorithm NETGEN2D in the SALOME platform [26, we used $N_{x}=150$ segments in the longitudinal direction, $N_{y}=45$ segments in the lateral direction and $N_{r}=20$ segments around the circumference of the cylinder. This makes, for the direction of wave propagation, a discretization of approximately 30 nodes per wavelength. In addition, $N_{z}=10$ nodes are used in the vertical direction on the tank sides and $N_{z}^{*}=30$ nodes are used for the vertical discretization of the cylinder. Incident wave conditions are imposed using the fifth-order Stokes theory. Fourier coefficients of the potential up to fourth-order are represented on Fig. 16, where they are compared to the experiments carried out by Huseby and Grue [37. Results match the experiments well, with a slight difference seen in the second harmonic, but this is quite consistent with other fully nonlinear computations, such as those by Ferrant [1] and Shao and Faltinsen 9]. Obtaining such an agreement for harmonics of the wave force signal up to the fourthorder clearly demonstrates the nonlinear capabilities of the proposed modeling approach. Note that contrary to Ferrant [1, we do not decompose the velocity potential into an incident component plus a diffraction component.

Note that the simulations performed here lead to simulated values for the forces which vary smoothly in time. The free surface pattern, can be noisy near the waterline without the filtering described by Eq. 40. As this test case is for a fixed body, we do not need to invoke the Laplace smoothing or regridding described earlier, simply this filtering along the waterline. Recent experimental tests of interactions between regular waves and vertical cylinders [38], however, suggest that waves near the waterline may physically be causing local breaking, or be damped by viscous effects. Calibrating the amount of dissipation required will be further investigated in future work; here we simply remove high-frequency waves, similar to Longuet-Higgins and Cokelet [39, which does not significantly affect waves which are well-resolved. 

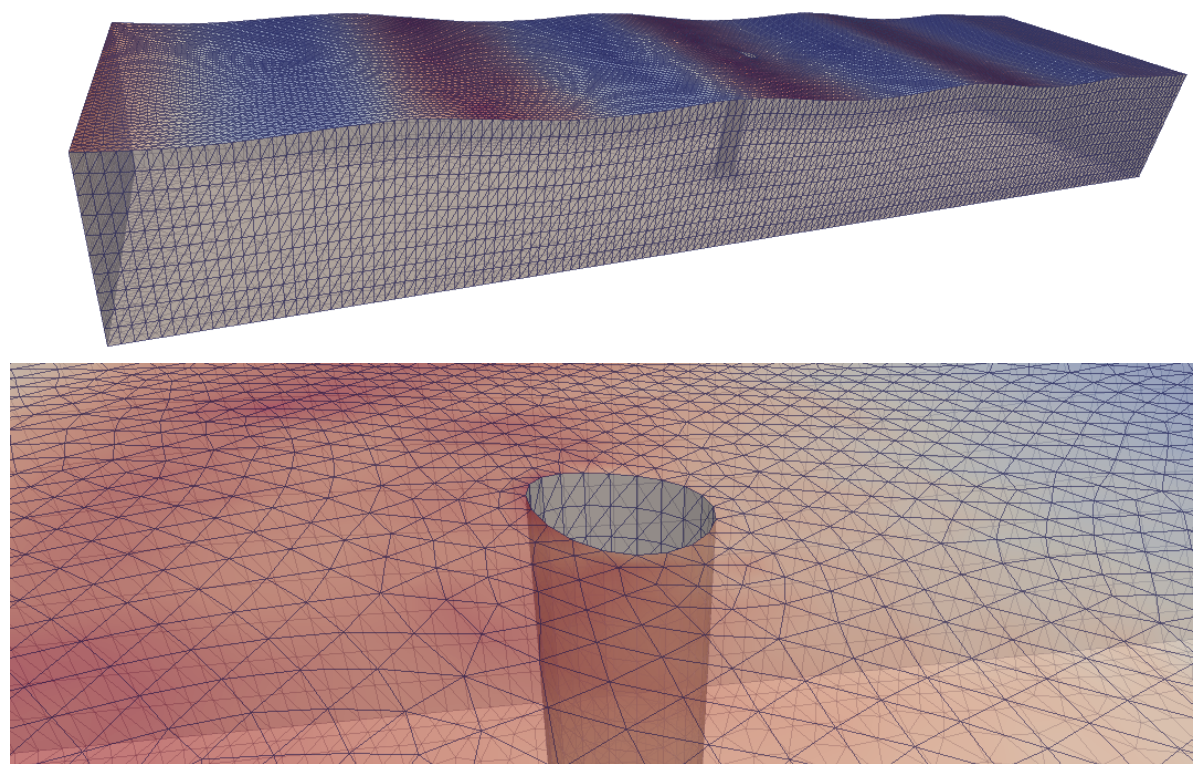

Figure 15: Computational domain (upper panel) and close-up of grid near cylinder (lower panel) for test cases of waves interacting with a fixed cylinder, in the case of $k R=0.245$ and $k A=0.10$.

324 4.3. Diffraction on a modified fixed Dutch Tri-floater platform

325 4.3.1. Setup of the model

We now consider a more complicated test case with a semi-submersible structure, 327 hereafter referred to as the Dutch Tri-floater [40, which is a proposed structure for 328 offshore wind turbines. The shape of the floater involves three surface piercing cylinders, 329 rigidly connected. Moreover, each cylinder is equipped at its base with a heave plate. 3зо This geometry is a slight simplification of original the Dutch Tri-floater geometry 40, ${ }_{331}$ as we do not consider here the smaller supporting struts, but they should not constitute 332 a significant source of hydrodynamic forces with an inviscid model. A sketch of the 333 geometry adopted in this work, also studied by Antonutti et al. in 41, is represented 334 on Fig. (17).

For the numerical wave tank, we again use a cylindrical domain such as the one represented in Fig. (6). This time, the reference free surface elevation $\eta^{r e f}$ and the reference potential $\phi^{r e f}$ correspond to the solution of the fifth-order Stokes theory. The

\begin{tabular}{lc}
\hline Design draft $(\mathrm{m})$ & 12.0 \\
Column centre-to-center spacing $(\mathrm{m})$ & 68.0 \\
Column diameter $(\mathrm{m})$ & 8.0 \\
Column depth including plate $(\mathrm{m})$ & 24.0 \\
Plate diameter $2 r(\mathrm{~m})$ & 18.0 \\
Plate thickness $(\mathrm{m})$ & 1.0 \\
\hline
\end{tabular}

Table 2: Geometric parameters of the Dutch Tri-floater chosen as in the study 41. 

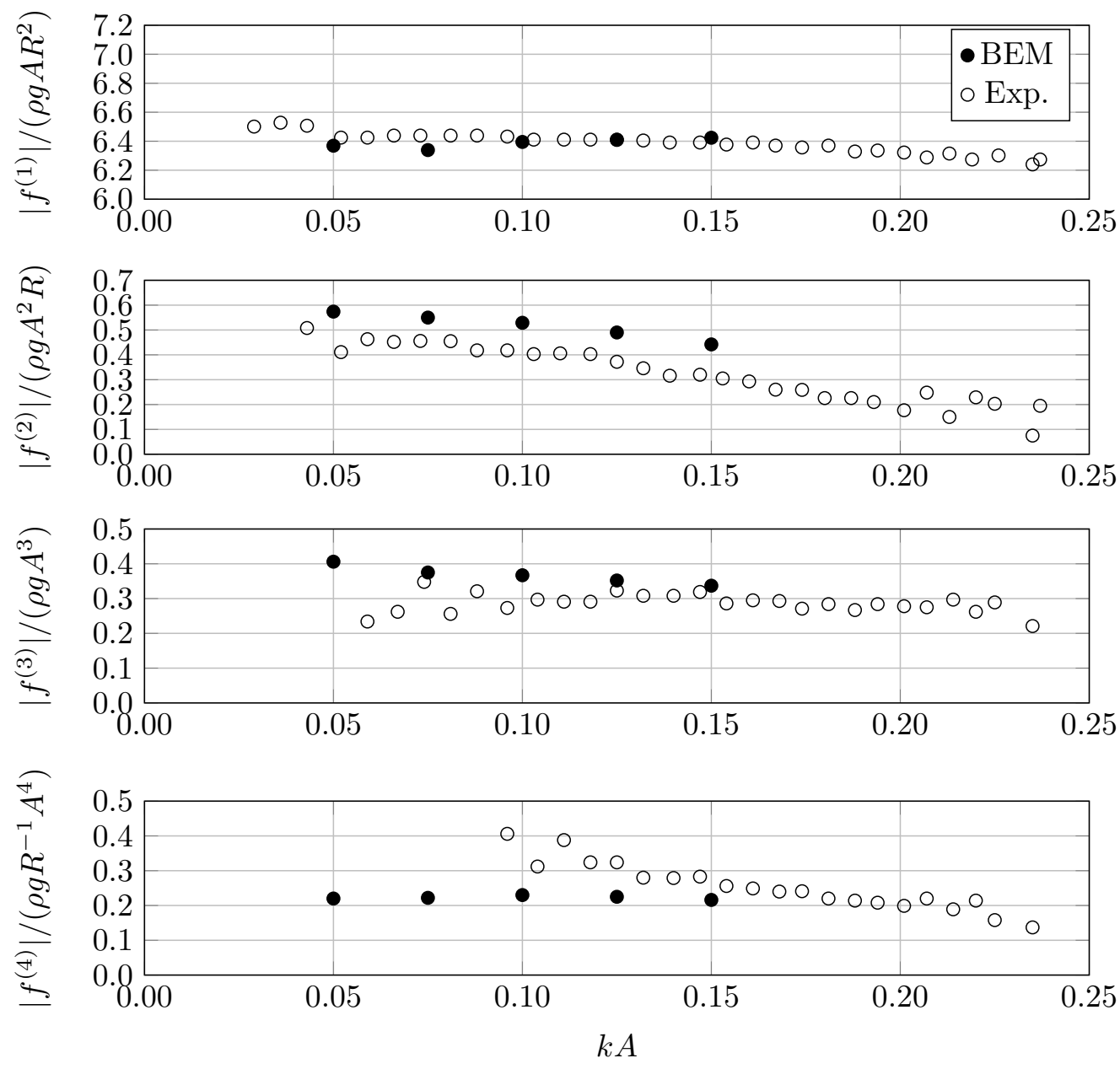

Figure 16: First through fourth-order Fourier coefficients associated to the time-series of $F_{x}$ for a bottommounted cylinder with a period $T=2.03 \mathrm{~s}, k R=0.245$ and the water depth $k d=\pi$.

coefficients requested to evaluate this solution are computed at the beginning of the simulation.

For the size of the domain, trial and error showed that a choice of parameters $R_{e x t}=$ $3 \lambda+\Lambda, R_{\lambda}=\lambda+\Lambda$ with $\Lambda=70 \mathrm{~m}$ and $\gamma_{0}=1$, leads to a stable value of wave loads after a duration of 40 wave periods, except for nondimensional wavenumbers $k r \geq 2.5$, for which longer time simulations are needed. On the contrary, by choosing $\left(R_{\lambda}, R_{e x t}\right)=$ $(2 \lambda+\Lambda, 3 \lambda+\Lambda)$ or $\left(R_{\lambda}, R_{e x t}\right)=(\lambda+\Lambda, 2 \lambda+\Lambda)$, that is, with an absorbing beach of radial length $\lambda$, we cannot obtain a convergence of the wave loads towards a periodic state.

We study below the diffraction of monochromatic waves on the floater. The waves are generated by imposing the velocity and acceleration of fifth-order Stokes waves on the far-field lateral sides. In addition, as already mentioned earlier, the wave profile 

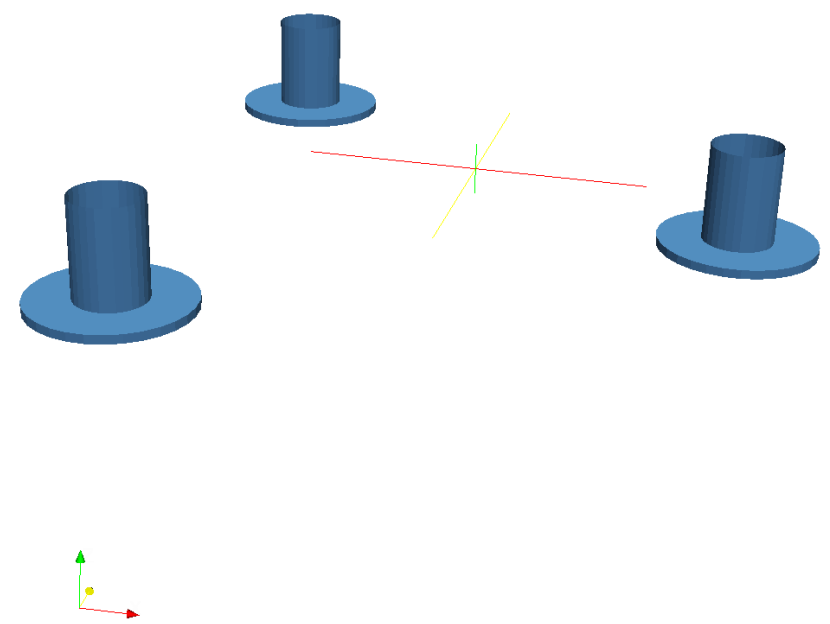

Figure 17: Geometry of the modified Dutch Tri-floater taken into account in the potential flow model. Above the mean free surface level, each cylinder is assumed to be infinite.

is damped out near the external boundary. With the methodology exposed above, we were able to simulate successfully the waves whose characteristics are given in Table (3) of the Appendix A. We note that for the steepest waves, the convergence of the wave loads toward a periodic state is slower, which suggests that in the future, it could be worth computing the Response Amplitude Operator (RAO) curve with a fixed steepness rather than with a fixed wave height (as done here in the traditional manner). The meshes used in this study are always adapted to the wavelength under consideration. The unstructured mesh of the free surface is done with the algorithm NETGEN2D in the SALOME platform [26. Each simulation used a mesh with a number of degrees of freedom in the range: $N_{d o f} \in \llbracket 18000,23000 \rrbracket$.

\subsubsection{Zero and first order loads}

We analyze the time-series of the forces $F_{x}$ and $F_{y}$, and the overturning moment $M_{y}$, over a window of 6 periods. Details on the time intervals considered for the Fourier analysis are given in Table 3 of Appendix A. For each frequency, we define the RAO of the temporal signal $F$ as the ratio between the first-order Fourier coefficient $F^{(1)}$ and the wave height $H$, and we assume that the density of the water is $\rho_{f}=1025 \mathrm{~kg} / \mathrm{m}^{3}$.

The RAO of the longitudinal force, $F_{x}$, agrees very well with the open-source linear wave model NEMOH [42] (Fig. 18). In particular, we find that the oscillations of the curve are well reproduced for the small periods. Similar agreements are found for the vertical force (Fig. 19), using reference values published in 41]. There are some deviations between the two results, even for these moderate wave amplitudes, at the peaks, as one might expect between linear and nonlinear models. Similarly, the nonlinear model described here predicts a lower maximum overturning moment, as compared to the linear model NEMOH (Fig. 20). 
374 In addition, we show in Fig. (21) the horizontal drift force, defined as the zero-order 375 coefficient $F_{x}^{(0)}$ of the Fourier series $F_{x}$, with respect to the wave period $T$. Our results are 376 compared to a frequency model computing the Quadratic Transfer Functions described

377 in 43 . Except for the smallest periods, a very good agreement with the weakly nonlinear 378 model is observed. For the highest frequencies tested in this study, our semi-Lagrangian scheme exhibits important deviations from the theory. This calculation provides further confidence in the ability of our numerical model to represent nonlinear features of the wave-structure interaction problem.

As an illustration of the importance of nonlinear effects, we choose to represent on Fig. (22), the ratio of second-order to first-order coefficients for the quantities of interest: $F_{x}, F_{z}$ and $M_{y}$. Whereas this ratio does not exceed $20 \%$ for $F_{x}$, it may reach $60 \%$ for $F_{z}$ and $M_{y}$, for the smallest periods of this study. This shows that nonlinearities become important for these shorter wavelengths with the Dutch Tri-floater.

\section{Conclusions}

We presented in this paper an implementation of a fully nonlinear potential wave model to simulate wave-structure interactions using unstructured triangular meshes, important for being able to handle future industrial applications with arbitrary problem geometry. The assembling of the system matrix is made with an efficient use of parallelization on distributed computer systems. Two time-stepping schemes, based on discrete derivatives with first-order shape functions, are derived. The accuracy of the whole algorithm could be easily enhanced by using high-order elements.

The model is applied to various problems involving surface piercing cylinders. The forces on a truncated cylinder in finite water depth, subjected to a prescribed sway motion, using a fully Lagrangian motion of the free surface mesh, is found to agree reasonably well with reference results. Similarly, forces on a bottom-mounted vertical cylinder resulting from diffraction of regular waves, using a semi-Lagrangian time-stepping scheme, capture higher-order nonlinear effects (i.e., up to the fourth harmonic of the horizontal force).

Finally, in order to show the potential of the method in dealing with complex structures, we also successfully compute both linear diffraction loads and nonlinear drift forces for a geometry inspired by the Dutch Tri-floater [40. Other important features, such as improving computational speed (i.e., with the fast multipole method), such as started by Harris et al. 44, will be considered in upcoming works, as well as using improved accuracy (i.e., cubic B-spline elements), or including more physics (i.e., coupling to NavierStokes solvers). Moreover, the case of arbitrary geometries for the rigid body needs to be addressed.

\section{Acknowledgements}

The contribution of E. Dombre to this work was partly funded by the French ANRT Agency (CIFRE agreement \# 2011-1724) and by the Fondation des Ponts through the Barré de Saint-Venant research fund. The work of J. C. Harris and M. Benoit was partially funded as a part of the French ANR (Agence Nationale de la Recherche), project ANR11-MONU-018-01 MONACOREV. 


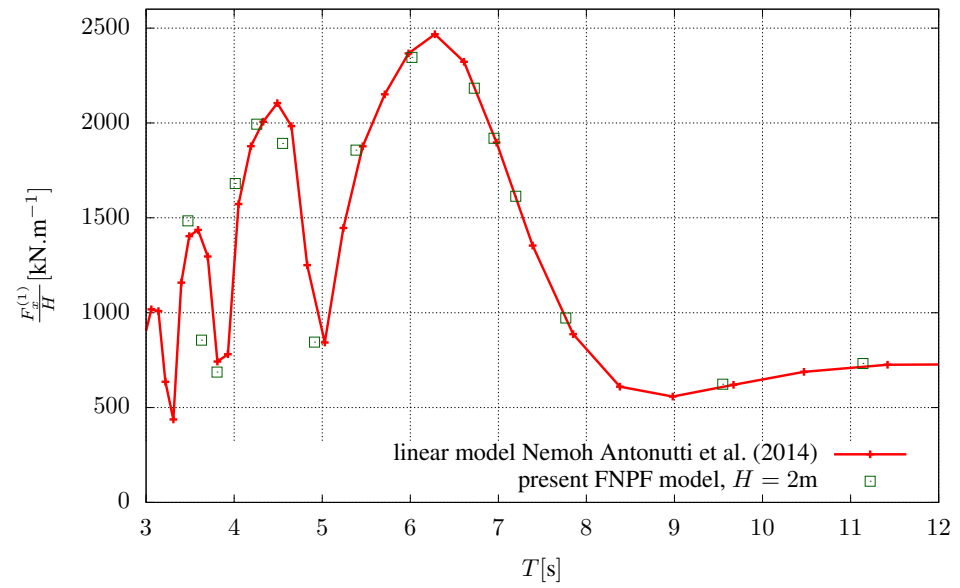

Figure 18: Nonlinear diffraction of a wave of height $H=2 \mathrm{~m}$ around the modified Dutch Tri-floater for various periods $T$ with a water depth of $d=50 \mathrm{~m}$ : RAO of the horizontal force $F_{x}$.

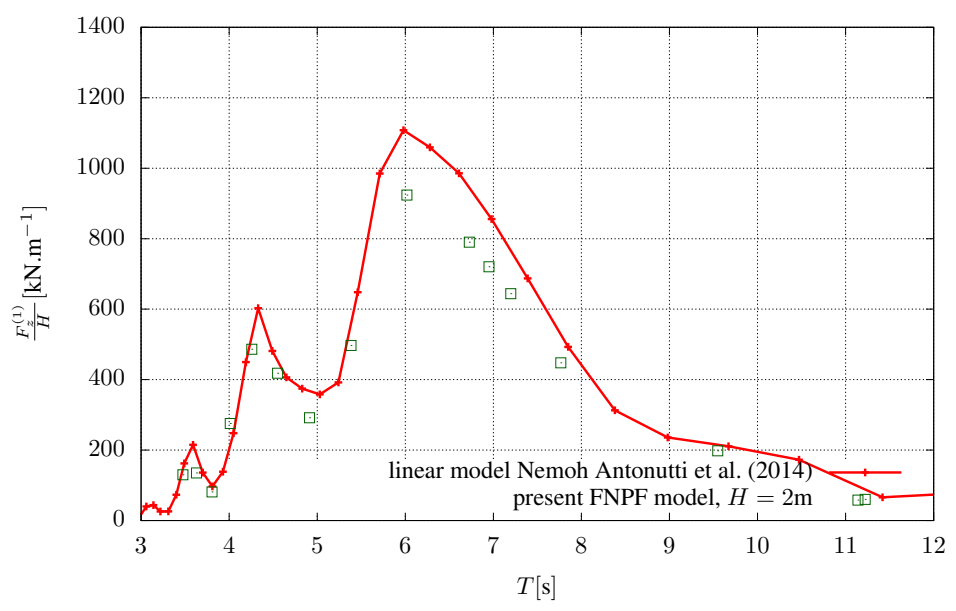

Figure 19: Nonlinear diffraction of a wave of height $H=2 \mathrm{~m}$ around the modified Dutch Tri-floater for various periods $T$ with a water depth of $d=50 \mathrm{~m}$ : RAO of the vertical force $F_{z}$. 


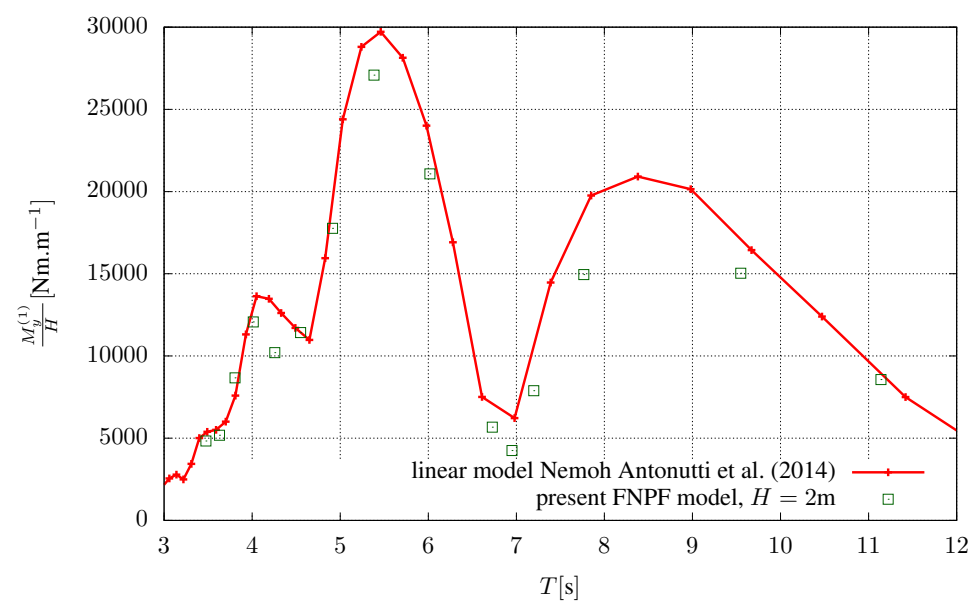

Figure 20: Nonlinear diffraction of a wave of height $H=2 \mathrm{~m}$ around the modified Dutch Tri-floater for various periods $T$ with a water depth of $d=50 \mathrm{~m}$ : RAO of the overturning moment $M_{y}$.

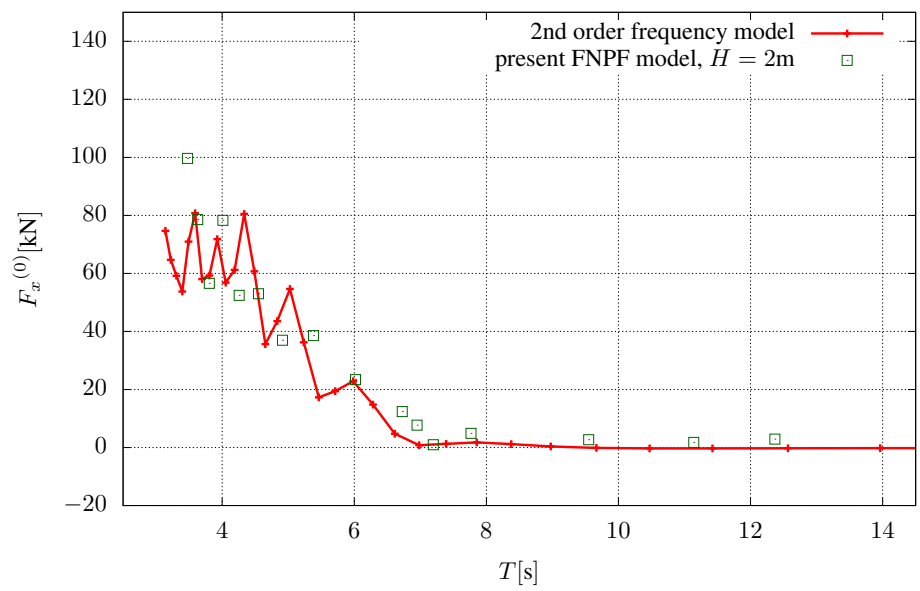

Figure 21: Nonlinear diffraction of a wave of height $H=2 \mathrm{~m}$ around the modified Dutch Tri-floater for various periods $T$ with a water depth of $d=50 \mathrm{~m}$ : horizontal drift force $F_{x}{ }^{(0)}$. 

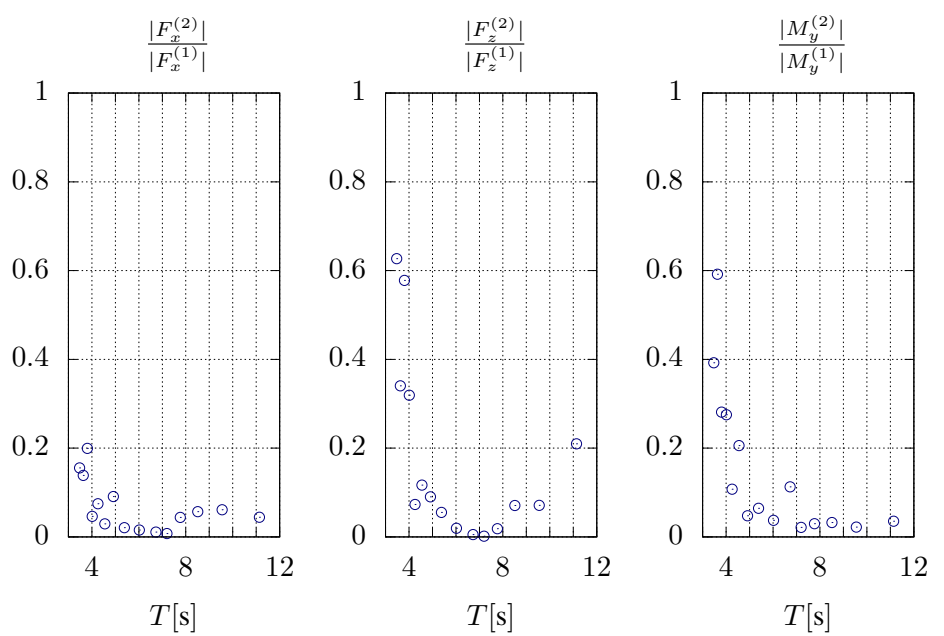

Figure 22: Nonlinear diffraction of a wave of height $H=2 \mathrm{~m}$ around the modified Dutch Tri-floater: ratio between second-order to first-order coefficients for the quantities $F_{x}, F_{z}$ and $M_{y}$.

416 Appendix A. Characteristics of the waves and the wave load analysis for the nonlinear diffraction around the modified Dutch Tri-floater.

418 In Table (3), the parameters of the waves used in the model and the time-interval of 419 the Fourier analysis are detailed for the test-case of the Dutch Tri-floater.

\begin{tabular}{l|ccc}
\hline$k r$ & $\omega[\mathrm{rad} / \mathrm{s}]$ & $\frac{2 \pi H}{g T^{2}}$ & {$\left[t_{1}^{+}, t_{2}^{+}\right]$} \\
\hline 0.3 & 0.564 & 0.010 & {$[13.47,19.47]$} \\
0.4 & 0.658 & 0.014 & {$[15.71,21.71]$} \\
0.6 & 0.809 & 0.021 & {$[12.88,18.88]$} \\
0.7 & 0.873 & 0.025 & {$[13.90,19.90]$} \\
0.75 & 0.904 & 0.027 & {$[14.39,20.39]$} \\
0.8 & 0.934 & 0.028 & {$[16.35,22.35]$} \\
1.0 & 1.044 & 0.036 & {$[24.93,30.93]$} \\
1.25 & 1.167 & 0.044 & {$[24.15,30.15]$} \\
1.50 & 1.279 & 0.053 & {$[24.43,30.43]$} \\
1.75 & 1.381 & 0.063 & {$[26.38,32.38]$} \\
2.0 & 1.476 & 0.071 & {$[29.36,35.36]$} \\
2.25 & 1.566 & 0.080 & {$[31.16,37.16]$} \\
2.50 & 1.651 & 0.089 & {$[63.07,69.07]$} \\
2.75 & 1.731 & 0.098 & {$[63.37,69.37]$} \\
3.00 & 1.808 & 0.108 & {$[63.31,69.31]$} \\
\hline
\end{tabular}

Table 3: Nonlinear diffraction for a wave height $H=2 \mathrm{~m}$ around the modified Dutch Tri-floater: characteristics of the waves simulated with respect to the non-dimensional wavenumber. The parameter $r$ denotes the radius of the heave plates and is set to $r=9 \mathrm{~m}$. $d$ is the water-depth, such that $\frac{H}{d}=0.04$. $\omega$ is the angular wave frequency. $k$ is the wave number. $T$ is the wave period. $t_{1}^{+}=\frac{t_{1}}{T}$ and $t_{2}^{+}=\frac{t_{2}}{T}$ with $\left[t_{1}, t_{2}\right]$ the time interval on which the Fourier analysis is performed. 


\section{References}

[1] P. Ferrant, Fully nonlinear interactions of long-crested wave packets with a three dimensional body, in: Proceedings of Twenty-Second ONR Symposium in Naval Hydrodynamics, 1998, pp. 59-72.

[2] S. Boo, Linear and nonlinear irregular waves and forces in a numerical wave tank, Ocean Engineering 29 (5) (2002) 475-493.

[3] Y. Liu, M. Xue, D. K. P. Yue, Computations of fully nonlinear three-dimensional wave-wave and wave-body interactions. Part 2. Nonlinear waves and forces on a body, Journal of Fluid Mechanics 438 (2001) 41-66.

[4] Š. Malenica, B. Molin, Third-harmonic wave diffraction by a vertical cylinder, Journal of Fluid Mechanics 302 (1995) 203-229.

[5] W. Bai, R. Eatock Taylor, Higher-order boundary element simulation of fully nonlinear wave radiation by oscillating vertical cylinders, Applied Ocean Research 28 (4) (2006) 247-265.

[6] W. Bai, R. Eatock Taylor, Fully nonlinear simulation of wave interaction with fixed and floating flared structures, Ocean Engineering 36 (3) (2009) 223-236.

[7] Q. Ma, G. Wu, R. Eatock Taylor, Finite element simulation of fully non-linear interaction between vertical cylinders and steep waves. Part 1: methodology and numerical procedure, International Journal for Numerical Methods in Fluids 36 (3) (2001) 265-285.

[8] C. Wang, G. Wu, K. Drake, Interactions between nonlinear water waves and non-wall-sided 3D structures, Ocean engineering 34 (8) (2007) 1182-1196.

[9] Y.-L. Shao, O. Faltinsen, A Harmonic polynomial cell (HPC) method for 3D Laplace equation with application in marine hydrodynamics, Journal of Computational Physics 274 (2014) 312-332.

[10] A. Mola, L. Heltai, A. DeSimone, A stable and adaptive semi-lagrangian potential model for unsteady and nonlinear ship-wave interactions, Engineering Analysis with Boundary Elements 37 (1) (2013) 128-143.

[11] S. Yan, Q. Ma, Numerical simulation of fully nonlinear interaction between steep waves and 2D floating bodies using the QALE-FEM method, Journal of Computational Physics 221 (2) (2007) $666-692$.

[12] C. Wang, G. Wu, An unstructured-mesh-based finite element simulation of wave interactions with non-wall-sided bodies, Journal of Fluids and Structures 22 (4) (2006) 441-461.

[13] G. Wu, Z. Hu, Simulation of nonlinear interactions between waves and floating bodies through a finite-element-based numerical tank, Proceedings of the Royal Society of London. Series A: Mathematical, Physical and Engineering Sciences 460 (2050) (2004) 2797-2817.

[14] Q. Ma, S. Yan, QALE-FEM for numerical modelling of non-linear interaction between 3D moored floating bodies and steep waves, International Journal for Numerical Methods in Engineering 78 (6) (2009) $713-756$.

[15] D. C. Kring, F. T. Korsmeyer, J. Singer, D. Danmeier, J. White, Accelerated nonlinear wave simulations for large structures, in: 7th International Conference on Numerical Ship Hydrodynamics, Nantes, France, 1999.

[16] S. T. Grilli, P. Guyenne, F. Dias, A fully non-linear model for three-dimensional overturning waves over an arbitrary bottom, International Journal for Numerical Methods in Fluids 35 (7) (2001) $829-867$.

[17] R. Machane, E. Canot, High-order schemes in boundary element methods for transient non-linear free surface problems, International Journal for Numerical Methods in Fluids 24 (1997) 1049-1072.

[18] E. Dombre, M. Benoit, D. Violeau, C. Peyrard, S. T. Grilli, Simulation of floating structure dynamics in waves by implicit coupling of a fully non-linear potential flow model and a rigid body motion approach, Journal of Ocean Engineering and Marine Energy 1 (1) (2015) 55-76.

[19] S. T. Grilli, R. Subramanya, A computer program for transient wave run-up, Tech. Rep. CACR93-02, Department of Ocean Engineering, University of Rhode Island (1993).

[20] D. Dunavant, High degree efficient symmetrical Gaussian quadrature rules for the triangle, International journal for numerical methods in engineering 21 (6) (1985) 1129-1148.

[21] W. Bai, B. Teng, Simulation of second-order wave interaction with fixed and floating structures in time domain, Ocean Engineering 74 (2013) 168-177.

[22] S. T. Grilli, I. A. Svendsen, Corner problems and global accuracy in the boundary element solution of nonlinear wave flows, Engineering Analysis with Boundary Elements 7 (4) (1990) 178-195.

[23] J. Dold, An efficient surface-integral algorithm applied to unsteady gravity waves, Journal of Computational Physics 103 (1) (1992) 90-115.

[24] J. Zhang, M. Kashiwagi, Application of ALE to nonlinear wave diffraction by a non-wall-sided 


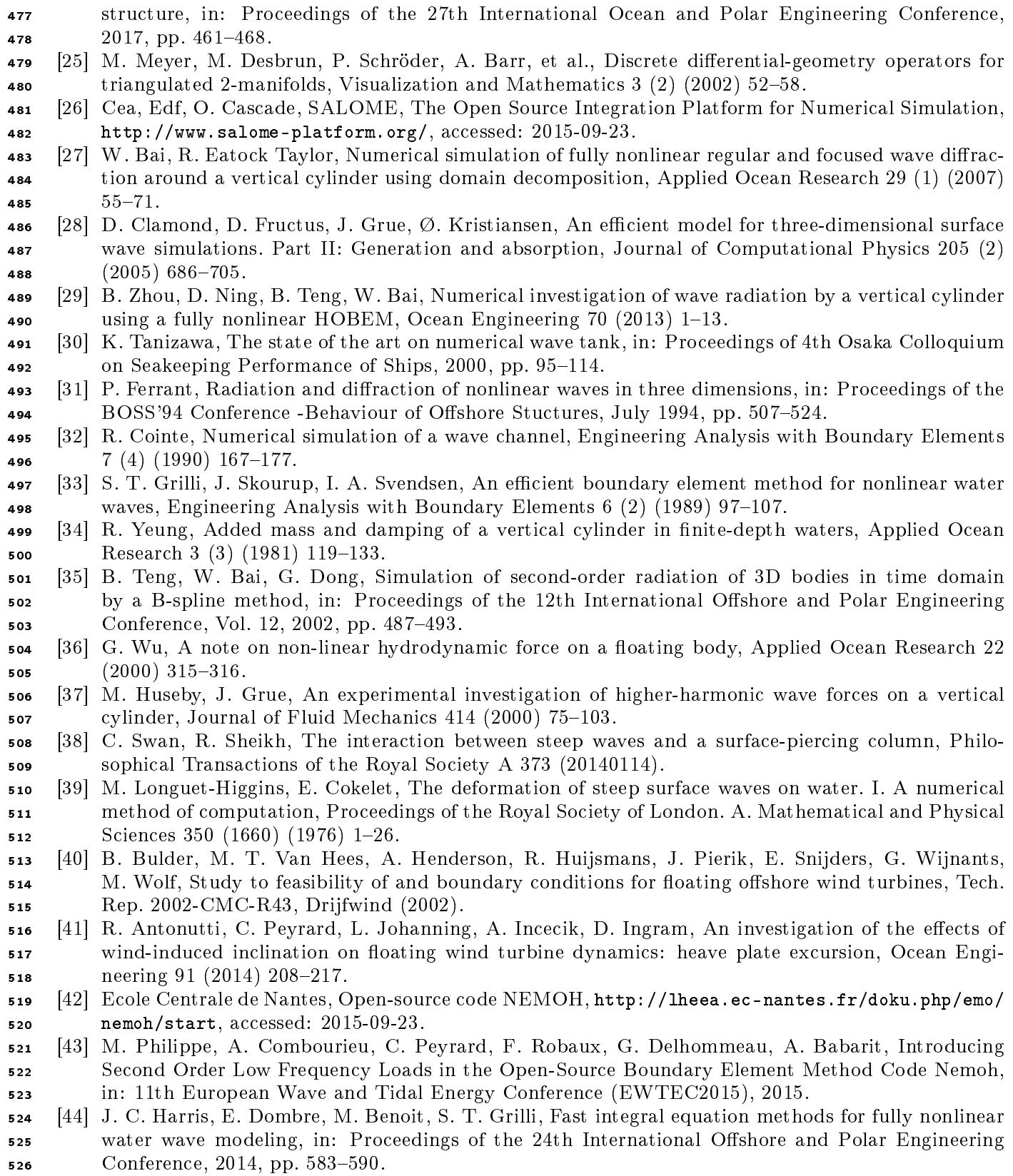

